\title{
THE NONLINEAR MEMBRANE MODEL: A YOUNG MEASURE AND VARIFOLD FORMULATION
}

\author{
Med Lamine Leghmizi ${ }^{1}$, Christian Licht $^{2}$ And Gérard Michaille ${ }^{3}$
}

\begin{abstract}
We establish two new formulations of the membrane problem by working in the space of $W_{\Gamma_{0}}^{1, p}\left(\Omega, \mathbf{R}^{3}\right)$-Young measures and $W_{\Gamma_{0}}^{1, p}\left(\Omega, \mathbf{R}^{3}\right)$-varifolds. The energy functional related to these formulations is obtained as a limit of the $3 d$ formulation of the behavior of a thin layer for a suitable variational convergence associated with the narrow convergence of Young measures and with some weak convergence of varifolds. The interest of the first formulation is to encode the oscillation informations on the gradients minimizing sequences related to the classical formulation. The second formulation moreover accounts for concentration effects.
\end{abstract}

Mathematics Subject Classification. 74K15, 35B05, 49J45.

Received February 11, 2004.

\section{INTRODUCTION}

Let $\omega$ be an open bounded subset of $\mathbf{R}^{2}$ and consider a thin layer of size $\varepsilon>0$, whose reference configuration $\Omega_{\varepsilon}=\omega \times(0, \varepsilon)$ is filled up by some elastic material. The structure is assumed to be clamped on $\Gamma_{0, \varepsilon}=\gamma_{0} \times(0, \varepsilon)$ where $\gamma_{0}$ is a part of the boundary of $\omega$ with non null one dimensional Lebesgue measure.

The stored strain energy associated with a deformation field $u: \Omega_{\varepsilon} \longrightarrow \mathbf{R}^{3}$ is given by an integral functional

$$
F_{\varepsilon}(u)=\int_{\Omega_{\varepsilon}} f(\nabla u) \mathrm{d} x
$$

associated with an elastic density function $f$ satisfying classical growth conditions. The equilibrium configuration of the structure is given by the optimization problem:

$$
\left(\mathcal{P}_{\varepsilon}\right) \quad \inf \left\{F_{\varepsilon}(u)-\int_{\Omega_{\varepsilon}} g_{\varepsilon} \cdot u \mathrm{~d} x: u \in W_{\Gamma_{0, \varepsilon}}^{1, p}\left(\Omega_{\varepsilon}, \mathbf{R}^{3}\right)\right\}
$$

where

$$
L_{\varepsilon}(u)=\int_{\Omega_{\varepsilon}} g_{\varepsilon} \cdot u \mathrm{~d} x
$$

Keywords and phrases. Membrane, Young measures, varifolds.

1 Centre Universitaire de Médéa, Institut des Sciences de l'Ingénieur, CC 151, Quartier Ain-D’Heb, Médéa (26000), Algeria.

2 Laboratoire de Mécanique et de Génie Civil, UMR-CNRS 5508, Université Montpellier II, Case courier 048, Place Eugène Bataillon, 34095 Montpellier Cedex 5, France.

3 ACSIOM et EMIAN, UMR-CNRS 5149, Université Montpellier 2 et CUFR de Nîmes, Case courier 051, Place Eugène Bataillon, 34095 Montpellier Cedex 5, France. 
denotes the exterior loading associated with applied body forces $g_{\varepsilon}$ living in $L^{q}\left(\Omega_{\varepsilon}, \mathbf{R}^{3}\right)$, and $W_{\Gamma_{0, \varepsilon}}^{1, p}\left(\Omega_{\varepsilon}, \mathbf{R}^{3}\right)$ the space of all the functions of $W^{1, p}\left(\Omega_{\varepsilon}, \mathbf{R}^{3}\right)$ having a null trace on $\Gamma_{0, \varepsilon}$.

We assume that this mechanical structure is occupied by a material which undergoes reversible solid/solid phase transformations as for instance some cristalline solids, so that the elastic density energy $f$ may possess various potential wells and is not quasiconvex. Consequently the total energy presents a lack of lower semicontinuity related to the weak convergence on $W_{\Gamma_{0, \varepsilon}}^{1, p}\left(\Omega_{\varepsilon}, \mathbf{R}^{3}\right)$ and $\left(\mathcal{P}_{\varepsilon}\right)$ has no solution in general. Moreover, even if some lower semicontinuity hypothesis is imposed, due to the small parameter $\varepsilon$, the three dimensional problem $\left(\mathcal{P}_{\varepsilon}\right)$ is not well fitted to numerical treatment.

After rescalling the problem in order to work in the fixed Sobolev space $W_{\Gamma_{0}}^{1, p}\left(\Omega, \mathbf{R}^{3}\right), \Omega=\omega \times(0,1)$, equipped with its weak convergence, a classical procedure for obtaining a good formulation of the membrane problem, consists in computing the $\Gamma$-limit of a rescaled functional $\tilde{F}_{\varepsilon}$ when $\varepsilon$ goes to zero (see [9]). In our case, this strategy has the disadvantage to "quasiconvexify" the density of the limit functional, so that the limit problem does not provide information on the oscillations of gradients minimizing sequences of $\left(\mathcal{P}_{\varepsilon}\right)$ and does not account for "bidimensional" microstructures. More precisely, the limit energy is an integral functional whose density is the quasiconvexication of the function $f_{0}$ defined for every $3 \times 2$ matrix $\hat{\lambda}$, by $f_{0}(\hat{\lambda})=\inf \left\{f(\hat{\lambda}, \xi), \xi \in \mathbf{R}^{3}\right\}$.

An alternative way, described in this paper, consists in "enlarging" the space of admissible functions by considering a subspace of the set $\mathcal{Y}\left(\Omega ; \mathcal{M}^{3 \times 3}\right)$ of Young measures $\mu=\left(\mu_{x}\right)_{x \in \Omega} \otimes \mathcal{L}\left(\mu_{x}\right.$ is a probability measure on $\mathcal{M}^{3 \times 3}, \mathcal{L}$ is the Lebesgue measure on $\Omega$ ), well adapted to capture oscillations of the gradients. The functional $\tilde{F}_{\varepsilon}$ is written in term of Young measure argument and we compute a suitable "variational limit" of the new formulation when $\varepsilon$ goes to zero. The two limit problems give the same minimum energy value. Moreover there is some important connexions between them, as, for instance, the fact that each gradient Young solutions encodes oscillations of gradient minimizing sequences of $\left(\mathcal{P}_{\varepsilon}\right)$ and its barycenter field is the gradient of a solution of the classical limit problem. We would like to point out that the expression of the limit functional energy allow us to suggest a modeling which may account for microstructures in thin film. We recover the important result of Bhattacharya and James [3] which predicts the existence of exact untwinned austenite/martinsite interfaces.

In a second stage, we intend to capture possible concentration of gradients minimizing sequences $\left(\nabla u_{\varepsilon}\right)_{\varepsilon>0}$ of $\left(\mathcal{P}_{\varepsilon}\right)$. Following the previous idea, we introduce a new formulation of the membrane problem in term of $W^{1, p_{-}}$ varifolds, a recent concept introduced in [6] for analyzing oscillation and concentration effects. The basic idea is that concentrations of $\left(\nabla u_{\varepsilon}\right)_{\varepsilon>0}$ are encoded by the singular part of the weak limit of the measure $\left|\nabla u_{\varepsilon}\right|^{p} \mathcal{L}$ through the recession function $f_{0}^{\infty}$ of degree $p$ of the function $f_{0}$. Since $f_{0}^{\infty}(\widehat{\nabla} u) \hat{\mathcal{L}}=f_{0}^{\infty}(\widehat{\nabla} u /|\widehat{\nabla} u|)|\widehat{\nabla} u|^{p} \hat{\mathcal{L}}$, where $\hat{\nabla} u$ denotes the tangential gradient of $u$ and $\hat{\mathcal{L}}$ the Lebesgue measure on $\omega$, we are lead to also describe the functional $\tilde{F}_{\varepsilon}$ in term of the measures of the type $\left(\delta_{\frac{\nabla u}{|\nabla u|}(x)}\right)_{x \in \Omega} \otimes|\nabla u|^{p} \mathcal{L}$.

The paper is organized as follows. In Section 2, we recall the classical formulation of $\left(\mathcal{P}_{\varepsilon}\right)$ and the limit problem $(\mathcal{P})$. Section 3 is devoted to the functional analysis setting related to the Young measure and varifold formulations. In Section 4 we introduce the Young measure formulations of $\left(\mathcal{P}_{\varepsilon}\right)$ and the expected limit problem $(\mathcal{P})$. We establish the main Theorem 2 and its corollaries. Finally, in Section 5 we give a $W_{\Gamma_{0}}^{1, p}\left(\Omega, \mathbf{R}^{3}\right)$ varifold formulation and establish the main Theorem 3.

During the writing of this paper, we have been aware of the work of [7] which treats the membrane problem by capturing only oscillations in a quite similar way.

\section{The ClASSiCAL FORMUlation}

In order to take into account large purely elastic deformation, the constitutive law of the deformable body is associated to a non convex elastic density $f$ satisfying growth and continuity conditions of order $p>1$ : there exists three positive constants $\alpha, \beta, L$ such that

$$
\begin{aligned}
& \forall a \in \mathcal{M}^{3 \times 3}, \quad \alpha|a|^{p} \leq f(a) \leq \beta\left(1+|a|^{p}\right) \\
& \forall a, b \in \mathcal{M}^{3 \times 3}, \quad|f(a)-f(b)| \leq L|b-a|\left(1+|a|^{p-1}+|b|^{p-1}\right) .
\end{aligned}
$$


The stored strain energy associated with a deformation field $u: \Omega_{\varepsilon} \rightarrow \mathbf{R}^{3}$ is given by the integral functional $F: L^{p}\left(\Omega_{\varepsilon}, \mathbf{R}^{3}\right) \longrightarrow \mathbf{R} \cup\{+\infty\}$ defined by

$$
F_{\varepsilon}(u)=\left\{\begin{array}{l}
\frac{1}{\varepsilon} \int_{\Omega_{\varepsilon}} f(\nabla u) \mathrm{d} x \text { if } u \in W_{\Gamma_{0, \varepsilon}}^{1, p}\left(\Omega_{\varepsilon}, \mathbf{R}^{3}\right) \\
+\infty \text { otherwise. }
\end{array}\right.
$$

The scaling parameter $\varepsilon^{-1}$ accounts for the stiffness of the material. In the linearized elasticity framework, it corresponds to Lamé coefficients of order $\varepsilon^{-1}$.

The structure is subjected to applied body forces $g_{\varepsilon}: \Omega_{\varepsilon} \longrightarrow \mathbf{R}^{3}$ for which we make the following assumption: there exists a vector valued function $g: \Omega=\omega \times(0,1) \longrightarrow \mathbf{R}^{3}, g \in L^{q}\left(\Omega, \mathbf{R}^{3}\right)(1 / p+1 / q=1)$, such that $\varepsilon g_{\varepsilon}\left(\hat{x}, \varepsilon x_{3}\right)=g(x), x=\left(\hat{x}, x_{3}\right)$. The exterior loading is

$$
L_{\varepsilon}(u)=\int_{\Omega_{\varepsilon}} g_{\varepsilon} \cdot u \mathrm{~d} x
$$

so that the equilibrium configuration is given by the displacement vector fields $\bar{u}_{\varepsilon}$, solutions of the problem:

$$
\inf \left\{F_{\varepsilon}(u)-L_{\varepsilon}(u): u \in L^{p}\left(\Omega_{\varepsilon}, \mathbf{R}^{3}\right)\right\} .
$$

Due to the very small thickness $\varepsilon$ of the layer $\Omega_{\varepsilon}$, for computing an approximate equilibrium deformation field, it is illusory to make use of finite element method. The variational property of $\Gamma$-convergence would give us a new procedure: by letting $\varepsilon$ go to zero, to find a new (fictitious) material occupying the two dimensional membrane $\omega$ and to compute an approximate equilibrium displacement field by means of a two dimensional finite element method related to a discretization of the new structure. This scheme has been described in [9] and the limit problem is given by

where

$$
\inf \left\{F(u)-L(u): u \in L^{p}\left(\Omega, \mathbf{R}^{3}\right)\right\}
$$

$$
F(u)=\left\{\begin{array}{l}
\int_{\Omega} Q f_{0}(\widehat{\nabla} u) \mathrm{d} x \text { if } u \in W_{\Gamma_{0}}^{1, p}\left(\Omega, \mathbf{R}^{3}\right), \frac{\partial u}{\partial x_{3}}=0, \\
+\infty \text { otherwise }
\end{array}\right.
$$

$Q f_{0}$ denotes the quasiconvexification of the function $f_{0}$ defined by

$$
f_{0}(\hat{\lambda})=\inf \left\{(\hat{\lambda}, \xi), \xi \in \mathbf{R}^{3}\right\} .
$$

For the definition and main properties of the quasiconvexification we refer the reader to [4]. The functional $L$ is defined by

$$
L(u)=\int_{\omega} \bar{g} \cdot u \mathrm{~d} x, \quad \bar{g}(\hat{x})=\int_{0}^{1} g(\hat{x}, s) \mathrm{d} x .
$$

As said in introduction, we would like to moreover capture the oscillations and possible concentrations of gradient minimizing sequences of problems $\left(\mathcal{P}_{\varepsilon}\right)$ and we adopt the following strategy: we formulate the problem $\left(\mathcal{P}_{\varepsilon}\right)$ in terms of $W_{\Gamma_{0}}^{1, p}\left(\Omega, \mathbf{R}^{3}\right)$-Young measure or $W_{\Gamma_{0}}^{1, p}\left(\Omega, \mathbf{R}^{3}\right)$-varifold and we compute a suitable variational limit when $\varepsilon$ goes to zero.

In order to work in the fixed space $L^{p}\left(\Omega, \mathbf{R}^{3}\right), \Omega=\omega \times(0,1)$, the change of scale $\left(\hat{x}, x_{3}\right)=\left(\hat{x}, \varepsilon x_{3}^{\prime}\right)$ transforming $\left(\hat{x}, x_{3}\right) \in \Omega_{\varepsilon}$ into $\left(\hat{x}, x_{3}^{\prime}\right) \in \Omega$ leads to the following equivalent optimization problem:

$$
\inf \left\{\tilde{F}_{\varepsilon}(v)-\int_{\Omega} g \cdot v \mathrm{~d} x: v \in L^{p}\left(\Omega, \mathbf{R}^{3}\right)\right\}
$$


where

$$
\tilde{F}_{\varepsilon}(v)=\left\{\begin{array}{l}
\int_{\Omega} f\left(\widehat{\nabla} v, \frac{1}{\varepsilon} \frac{\partial v}{\partial x_{3}}\right) \mathrm{d} x \text { if } v \in W_{\Gamma_{0}}^{1, p}\left(\Omega, \mathbf{R}^{3}\right) \\
+\infty \text { otherwise }
\end{array}\right.
$$

$\Gamma_{0}=\gamma_{0} \times(0,1), \widehat{\nabla} v$ denotes the tangential gradient of $v$ defined by $\widehat{\nabla} v=\left(\frac{\partial v_{i}}{\partial x_{j}}\right)_{i=1,2,3, j=1,2}$.

Note that if the magnitude of the stiffness and of the forces are of order one, this result shows that the total energy of the structure is equivalent to $\varepsilon(F-L)$ in a variational sense.

All the results of the paper remain valid when working with a density $f$ dependaing on the plane variable $\hat{x}$, provided that growth conditions be uniform on $\hat{x}$.

\section{SOME SUITABLE SETS FOR CAPTURING OSCILLATIONS AND CONCENTRATION EFFECTS}

\subsection{Young measures and varifolds parametrized on $\Omega$}

We denote the sets of $3 \times 3$ and $3 \times 2$ matrices with real numbers entries by $\mathcal{M}^{3 \times 3}$ and $\mathcal{M}^{3 \times 2}$, respectively, and their unit spheres by $\mathcal{S}^{3 \times 3}$ and $\mathcal{S}^{3 \times 2}$. Considering the space $\mathcal{M}^{3 \times 3}$ as the product $\mathcal{M}^{3 \times 2} \times \mathbf{R}^{3}$, we will denote by $\hat{\lambda}$ the first coordinate in $\mathcal{M}^{3 \times 2}$ of any element $\lambda$ of $\mathcal{M}^{3 \times 3}$.

We define the sets of Young measures $\mathcal{Y}_{3 \times 3}(\Omega)$ and varifolds $\mathcal{Y} \mathcal{V}_{3 \times 3}(\Omega)$, parametrized on $\Omega$, as follows:

$$
\begin{aligned}
& \mu \in \mathcal{Y}_{3 \times 3}(\Omega) \Longleftrightarrow \mu \in \mathbf{M}^{+}\left(\Omega \times \mathcal{M}^{3 \times 3}\right) \text { and } P_{\Omega} \# \mu=\mathcal{L} \\
& \mathcal{Y} \mathcal{V}_{3 \times 3}(\Omega)=\mathcal{Y}_{3 \times 3}(\Omega) \times \mathbf{M}^{+}\left(\Omega \times \mathcal{S}^{3 \times 3}\right)
\end{aligned}
$$

where $P_{\Omega} \# \mu$ denotes the image of the measure $\mu$ by the projection $P_{\Omega}: \Omega \times \mathcal{M}^{3 \times 3} \rightarrow \Omega$.

We now define the space $\mathcal{C}_{b}\left(\Omega ; \mathcal{M}^{3 \times 3}\right)$ of all Carathéodory integrands, namely, the space of all measurable functions $\psi: \Omega \times \mathcal{M}^{3 \times 3} \rightarrow \mathbf{R}$ such that

i) $\psi(x,$.$) is bounded continuous on \mathcal{M}^{3 \times 3}$ for every $x \in \Omega$;

ii) $x \mapsto\|\psi(x, .)\|_{\infty}$ is Lebesgue integrable.

On the other hand, classically, we denote by $\mathcal{C}_{0}\left(\Omega \times \mathcal{S}^{3 \times 3}\right)$ the space of all continuous functions $\varphi: \Omega \times \mathcal{S}^{3 \times 3} \rightarrow \mathbf{R}$ vanishing on the boundary $\partial \Omega \times \mathcal{S}^{3 \times 3}$.

Let us recall that the narrow convergence of a sequence $\left(\mu_{n}\right)_{n \in \mathbf{N}}$ to $\mu$ in $\mathcal{Y}_{3 \times 3}(\Omega)$ and the weak convergence of a sequence $\left(\theta_{n}\right)_{n \in \mathbf{N}}$ to $\theta$ in $\mathbf{M}^{+}\left(\Omega \times \mathcal{S}^{3 \times 3}\right)$ are defined as follows:

$$
\mu_{n} \stackrel{\text { nar }}{\rightarrow} \mu \Longleftrightarrow \lim _{n \rightarrow+\infty} \int_{\Omega \times \mathcal{M}^{3 \times 3}} \psi(x, \lambda) \mathrm{d} \mu_{n}=\int_{\Omega \times \mathcal{M}^{3 \times 3}} \psi(x, \lambda) \mathrm{d} \mu
$$

for all $\psi \in \mathcal{C}_{b}\left(\Omega ; \mathcal{M}^{3 \times 3}\right)$,

$$
\theta_{n} \rightarrow \theta \Longleftrightarrow \lim _{n \rightarrow+\infty} \int_{\Omega \times \mathcal{S}^{3 \times 3}} \varphi(x, \lambda) \mathrm{d} \theta_{n}=\int_{\Omega \times \mathcal{S}^{3 \times 3}} \varphi(x, \lambda) \mathrm{d} \theta
$$

for all $\varphi \in \mathcal{C}_{0}\left(\Omega \times \mathcal{S}^{3 \times 3}\right)$.

In all the paper, we equip $\mathcal{Y}_{3 \times 3}(\Omega)$ with the narrow convergence denoted by $\stackrel{\text { nar }}{\longrightarrow}$ and $\mathcal{Y V}_{3 \times 3}(\Omega)$ with the product of the narrow convergence on $\mathcal{Y}_{3 \times 3}(\Omega)$ and the weak convergence on $\mathbf{M}^{+}\left(\Omega \times \mathcal{S}^{3 \times 3}\right)$ denoted by $\rightarrow$.

We now define the two following subspaces $E \nabla \mathcal{Y}_{3 \times 3}(\Omega)$ and $E \nabla \mathcal{Y} \mathcal{V}_{3 \times 3}(\Omega)$ of respectively $\mathcal{Y}_{3 \times 3}(\Omega)$ and $\mathcal{Y} \mathcal{V}_{3 \times 3}(\Omega)$ whose elements will be called respectively elementary $W_{\Gamma_{0}}^{1, p}\left(\Omega, \mathbf{R}^{3}\right)$-Young measures and elementary 
$W_{\Gamma_{0}}^{1, p}\left(\Omega, \mathbf{R}^{3}\right)$-varifolds:

$$
\begin{aligned}
& \mu \in E \nabla \mathcal{Y}_{3 \times 3}(\Omega) \Longleftrightarrow \exists u \in W_{\Gamma_{0}}^{1, p}\left(\Omega, \mathbf{R}^{3}\right), \mu=\left(\delta_{\nabla u(x)}\right)_{x \in \Omega} \otimes \mathcal{L}, \\
& (\mu, \theta) \in E \nabla \mathcal{Y} \mathcal{V}_{3 \times 3}(\Omega) \Longleftrightarrow\left\{\begin{array}{l}
\exists u \in W_{\Gamma_{0}}^{1, p}\left(\Omega, \mathbf{R}^{3}\right) \text { s.t.: } \\
\mu=\left(\delta_{\nabla u(x)}\right)_{x \in \Omega} \otimes \mathcal{L}, \\
\theta=\left(\delta_{\frac{\nabla u}{|\nabla u|}(x)}\right)_{x \in \Omega} \otimes|\nabla u|^{p} \mathcal{L} .
\end{array}\right.
\end{aligned}
$$

The Young measure $\mu$ and the varifold $(\mu, \theta)$ will be said to be associated with the function $u$. Note that if $(\mu, \theta)$ in $E \nabla \mathcal{Y} \mathcal{V}_{3 \times 3}(\Omega)$ is associated with $u$, then

$$
\int_{\Omega \times \mathcal{M}^{3 \times 3}} \psi(x, \lambda) \mathrm{d} \mu=\int_{\Omega} \psi(x, \nabla u) \mathrm{d} x
$$

and

whenever each integral exists.

$$
\int_{\Omega \times \mathcal{S}^{3 \times 3}} \psi(x, \lambda) \mathrm{d} \theta=\int_{\Omega} \psi\left(x, \frac{\nabla u}{|\nabla u|}\right)|\nabla u|^{p} \mathrm{~d} x
$$

We denote the sequential closures of $E \nabla \mathcal{Y}_{3 \times 3}(\Omega)$ and $E \nabla \mathcal{Y V}_{3 \times 3}(\Omega)$, respectively, by $\nabla \mathcal{Y}_{3 \times 3}(\Omega)$ and $\nabla \mathcal{Y} \mathcal{V}_{3 \times 3}(\Omega)$. Their elements are respectively called $W_{\Gamma_{0}}^{1, p}\left(\Omega, \mathbf{R}^{3}\right)$-Young measures and $W_{\Gamma_{0}}^{1, p}\left(\Omega, \mathbf{R}^{3}\right)$-varifolds. A sequence $\left(u_{\varepsilon}\right)_{\varepsilon>0}$ whose associated sequence $\left(\left(\mu_{\varepsilon}, \theta_{\varepsilon}\right)\right)_{\varepsilon>0}$ of elementary $W_{\Gamma_{0}}^{1, p}\left(\Omega, \mathbf{R}^{3}\right)$-varifolds tends to a $W_{\Gamma_{0}}^{1, p}\left(\Omega, \mathbf{R}^{3}\right)$-varifold $(\mu, \theta)$ will be called generating the couple $(\mu, \theta)$.

Let $(\mu, \theta)$ be a $W_{\Gamma_{0}}^{1, p}\left(\Omega, \mathbf{R}^{3}\right)$-varifold generated by $\left(u_{\varepsilon}\right)_{\varepsilon>0}$ and let us denote by $\pi$ the image $\pi=P_{\Omega} \# \theta$ of $\theta$ by the projection $P_{\Omega}: \Omega \times \mathcal{S}^{3 \times 3} \rightarrow \Omega$. Obviously, $\pi$ is nothing but the weak limit of the measure $\left|\nabla u_{\varepsilon}\right|^{p} \mathcal{L}$.

According to the Slicing theorem (see [12,13] and Th. 4 of the Appendix), for every $(\mu, \theta)$ in $\mathcal{Y} \mathcal{V}_{3 \times 3}(\Omega)$, there exists a family of probability measures $\left(\mu_{x}\right)_{x \in \Omega}$ on $\mathcal{M}^{3 \times 3}$ and a family $\left(\theta_{x}\right)_{x \in \Omega}$ of probability measures on $\mathcal{S}^{3 \times 3}$ such that

$$
\begin{aligned}
& \mu=\left(\mu_{x}\right)_{x \in \Omega} \otimes \mathcal{L} \\
& \theta=(\theta)_{x \in \Omega} \otimes \pi .
\end{aligned}
$$

Let us consider the Lebesgue-Nikodym decomposition of the measure $\pi$ :

$$
\pi=\frac{\mathrm{d} \pi}{\mathrm{d} \mathcal{L}} \mathcal{L}+\pi_{s}
$$

We have the following characterization of $W_{\Gamma_{0}}^{1, p}\left(\Omega, \mathbf{R}^{3}\right)$-varifolds for $p>1$ (see [6]):

Theorem 1. A pair $(\mu, \theta)$ is a $W_{\Gamma_{0}}^{1, p}\left(\Omega, \mathbf{R}^{3}\right)$-varifold iff the four following assertions hold

(i) there exists $u \in W_{\Gamma_{0}}^{1, p}\left(\Omega, \mathbf{R}^{3}\right)$ such that $\nabla u(x)=\int_{\mathcal{M}^{3 \times 3}} \lambda \mathrm{d} \mu_{x}(\lambda), \mathcal{L}$ a.e. $x \in \Omega$;

(ii) $\psi(\nabla u(x)) \leq \int_{\mathcal{M}^{3 \times 3}} \psi(\lambda) \mathrm{d} \mu_{x}(\lambda)$, L a.e. $x \in \Omega$, for every quasiconvex function $\psi$ satisfying classical growth conditions of order $p$ :

$$
|\psi(\lambda)| \leq \beta\left(1+|\lambda|^{p}\right)
$$

(iii) $\int_{\mathcal{M}^{3 \times 3}} \psi(\lambda) \mathrm{d} \mu_{x}(\lambda) \leq \frac{\mathrm{d} \pi}{\mathrm{d} \mathcal{L}}(x) \int_{\mathcal{S}^{3 \times 3}} \psi(\lambda) \mathrm{d} \theta_{x}(\lambda), \mathcal{L}$ a.e. $x \in \Omega$, for every $p$-homogeneous continuous function $\psi$ such that $Q \psi(0)=0$, where $Q \psi$ denotes the quasiconvexification of $\psi$;

(iv) $\int_{\mathcal{S}^{3 \times 3}} \psi(\lambda) \mathrm{d} \theta_{x}(\lambda) \geq 0, \pi_{s}$ a.e. $x \in \Omega$ for every $p$-homogeneous continuous function $\psi$ such that $Q \psi(0)=0$. 


\section{Remark 1.}

1) Note that, using condition (iii) with $\psi(\lambda)=|\lambda|^{p}$ we obtain

$$
\int_{\Omega \times \mathcal{M}^{3 \times 3}}|\lambda|^{p} \mathrm{~d} \mu(x, \lambda)<+\infty
$$

and Theorem 1 then yields (i), (ii), (iii)', a well known characterization of $W_{\Gamma_{0}}^{1, p}\left(\Omega, \mathbf{R}^{3}\right)$-Young measures $\mu$ (see $[8,10,11])$.

2) The singular measure $\pi_{s}$ encodes concentrations effects of the sequence $\left(\nabla u_{\varepsilon}\right)_{\varepsilon>0}$ generating $\theta$ (see various examples in $[6])$.

The two following subspaces $\nabla \mathcal{Y}_{3 \times 2,0}(\Omega) \subset \nabla \mathcal{Y}_{3 \times 3}(\Omega)$ and $\nabla \mathcal{Y V}_{3 \times 2,0}(\Omega) \subset \nabla \mathcal{Y} \mathcal{V}_{3 \times 3}(\Omega)$ defined below will play an essential role in the Young measure and varifold formulation of the nonlinear membrane problem:

$$
\mu \in \nabla \mathcal{Y}_{3 \times 2,0}(\Omega) \Longleftrightarrow\left\{\begin{array}{l}
\exists\left(u_{\varepsilon}\right)_{\varepsilon>0} \text { bounded in } W_{\Gamma_{0}}^{1, p}\left(\Omega, \mathbf{R}^{3}\right) \text { s.t. } \\
\left(\delta_{\nabla u_{\varepsilon}(x)}\right)_{x \in \Omega} \otimes \mathcal{L} \stackrel{\text { nar }}{\rightarrow} \mu \\
\left(\delta_{\widehat{\nabla} u_{\varepsilon}(x)}\right)_{x \in \Omega} \otimes \mathcal{L} \stackrel{\text { nar }}{\rightarrow} \hat{\mu} \\
\mu_{x}=\hat{\mu}_{x} \otimes \delta_{0},
\end{array}\right.
$$

where $\left(\delta_{\hat{\nabla} u_{\varepsilon}(x)}\right)_{x \in \Omega} \otimes \mathcal{L} \stackrel{\text { nar }}{\longrightarrow} \hat{\mu}$ must be taken in the sense of the narrow convergence in the space $\mathcal{Y}_{3 \times 2}(\Omega)$ of Young measures parametrized on $\Omega$, with values in $\mathcal{M}^{3 \times 2}$ and $\left(\hat{\mu}_{x}\right)_{x \in \Omega}$ is the family of probability measures on $\mathcal{M}^{3 \times 2}$ stemming from the disintegration of the Young measure $\hat{\mu}$. To summarize, will write $\mu=\hat{\mu} \otimes \delta_{0}$;

$$
(\mu, \theta) \in \nabla \mathcal{Y} \mathcal{V}_{3 \times 2,0}(\Omega) \Longleftrightarrow\left\{\begin{array}{l}
\exists\left(u_{\varepsilon}\right)_{\varepsilon>0} \text { s.t. (3) holds and } \\
\left(\delta_{\frac{\nabla u_{\varepsilon}}{\mid \nabla u_{\varepsilon}}(x)}\right)_{x \in \Omega} \otimes|\nabla u|^{p} \mathcal{L} \rightarrow \theta \\
\left(\delta_{\widehat{\nabla} u_{\varepsilon}}(x)\right. \\
\left.\mid \frac{\nabla u_{\varepsilon} \mid}{\mid}\right)_{x \in \Omega} \otimes\left|\widehat{\nabla} u_{\varepsilon}\right|^{p} \mathcal{L} \rightarrow \hat{\theta} \\
P_{\Omega} \# \theta=P_{\Omega} \# \hat{\theta}:=\pi \\
\theta_{x}=H \# \hat{\theta}_{x} \pi \text { a.e. } x \in \Omega
\end{array}\right.
$$

where $\left(\delta_{\frac{\widehat{\nabla} u_{\varepsilon}}{\left|\nabla u_{\varepsilon}\right|}(x)}\right)_{x \in \Omega} \otimes\left|\widehat{\nabla} u_{\varepsilon}\right|^{p} \mathcal{L} \rightarrow \hat{\theta}$ must be taken in the sense of the weak convergence in $\mathbf{M}^{+}\left(\Omega \times \mathcal{S}^{3 \times 2}\right)$, $\left(\theta_{x}\right)_{x \in \Omega}$ and $\left(\hat{\theta}_{x}\right)_{x \in \Omega}$ are the familly of probability measures on the unit spheres $\mathcal{S}^{3 \times 3}$ and $\mathcal{S}^{3 \times 2}$ of $\mathcal{M}^{3 \times 3}$ and $\mathcal{M}^{3 \times 2}$, stemming from the desintegration of the measures $\theta$ and $\hat{\theta}$ with respect to $\pi$. The map $H$ is the extension operator $H: \mathcal{S}^{3 \times 2} \rightarrow \mathcal{S}^{3 \times 3}$ defined by $H(\hat{\lambda})=(\hat{\lambda}, 0)$.

It is worth noticing the similarity between (3) and (4). Indeed $\mu_{x}$ in (3) is nothing but $H \# \hat{\mu}_{x}$ if we extend $H$ into the map $\mathcal{M}^{3 \times 2} \rightarrow \mathcal{M}^{3 \times 3}, \hat{\lambda} \mapsto(\hat{\lambda}, 0)$.

\subsection{Young measures and varifolds parametrized on $\omega$}

Similarly we denote by $\mathcal{Y}_{3 \times 2}(\omega)$ and $\mathcal{Y V}_{3 \times 2}(\omega)$ the spaces of Young measures and varifolds parametrized on $\omega$, defined by:

$$
\begin{aligned}
& \mu \in \mathcal{Y}_{3 \times 2}(\omega) \Longleftrightarrow \mu \in \mathbf{M}^{+}\left(\omega \times \mathcal{M}^{3 \times 2}\right) \text { and } P_{\omega} \# \mu=\widehat{\mathcal{L}}, \\
& \mathcal{Y} \mathcal{V}_{3 \times 2}(\omega)=\mathcal{Y}_{3 \times 2}(\omega) \times \mathbf{M}^{+}\left(\omega \times \mathcal{S}^{3 \times 2}\right)
\end{aligned}
$$

where $\widehat{\mathcal{L}}$ denotes the Lebesgue measure on $\omega$ and $P_{\omega} \# \mu$ the image measure of $\mu$ by the projection $P_{\omega}: \omega \times$ $\mathcal{M}^{3 \times 2} \rightarrow \omega$. 
We equip $\mathcal{Y}_{3 \times 2}(\omega)$ with the narrow convergence and $\mathcal{Y V}_{3 \times 2}(\omega)$ with the product of the narrow convergence on $\mathcal{Y}_{3 \times 2}(\omega)$ and the weak convergence on $\mathbf{M}^{+}\left(\omega \times \mathcal{S}^{3 \times 2}\right)$.

We now define the subspaces of elementary $W_{\gamma_{0}}^{1, p}\left(\omega, \mathbf{R}^{3}\right)$-Young measures and elementary $W_{\gamma_{0}}^{1, p}\left(\omega, \mathbf{R}^{3}\right)$ varifolds as follows:

$$
\begin{aligned}
& \nu \in E \nabla \mathcal{Y}_{3 \times 2}(\omega) \Longleftrightarrow \exists u \in W_{\gamma_{0}}^{1, p}\left(\omega, \mathbf{R}^{3}\right), \nu=\left(\delta_{\nabla u(\hat{x})}\right)_{\hat{x} \in \omega} \otimes \widehat{\mathcal{L}}, \\
& (\nu, m) \in E \nabla \mathcal{Y} \mathcal{V}_{3 \times 2}(\omega) \Longleftrightarrow\left\{\begin{array}{l}
\exists u \in W_{\gamma_{0}}^{1, p}\left(\omega, \mathbf{R}^{3}\right) \text { s.t. : } \\
\nu=\left(\delta_{\nabla u(\hat{x})}\right) \hat{x} \in \omega \\
m=\left(\frac{\nabla u}{|\nabla u|}(\hat{x})\right)_{\hat{x} \in \omega} \otimes|\nabla u|^{p} \widehat{\mathcal{L}} .
\end{array}\right.
\end{aligned}
$$

The spaces $\nabla \mathcal{Y}_{3 \times 2}(\omega)$ and $\nabla \mathcal{Y} \mathcal{V}_{3 \times 2}(\omega)$ of $W_{\gamma_{0}}^{1, p}\left(\omega, \mathbf{R}^{3}\right)$-Young measures and $W_{\gamma_{0}}^{1, p}\left(\omega, \mathbf{R}^{3}\right)$-varifolds are the sequential closures of $E \nabla \mathcal{Y}_{3 \times 2}(\omega)$ and $E \nabla \mathcal{Y V}_{3 \times 2}(\omega)$ respectively. The analague of Theorem 1 holds with obvious adaptations.

\subsection{From parameters in $\Omega$ to parameters in $\omega$ : the mean operators $\Theta$ and $\Xi$}

We now define the two operators $\Theta$ from $\nabla \mathcal{Y}_{3 \times 2,0}(\Omega)$ into $\mathcal{Y}_{3 \times 2}(\omega)$ and $\Xi$ from $\nabla \mathcal{Y} \mathcal{V}_{3 \times 2,0}(\Omega)$ into $\mathcal{Y} \mathcal{V}_{3 \times 2}(\omega)$ as follows:

$$
\begin{gathered}
\Theta: \nabla \mathcal{Y}_{3 \times 2,0}(\Omega) \longrightarrow \mathcal{Y}_{3 \times 2}(\omega) \\
\mu=\hat{\mu} \otimes \delta_{0} \mapsto \overline{\hat{\mu}}, \text { with } \overline{\hat{\mu}}_{\hat{x}}:=\int_{0}^{1} \hat{\mu}_{\hat{x}, s} \mathrm{~d} x ; \\
\Xi: \nabla \mathcal{Y} \mathcal{V}_{3 \times 2,0}(\Omega) \longrightarrow \mathcal{Y} \mathcal{V}_{3 \times 2}(\omega) \\
(\mu, \theta) \mapsto(\overline{\hat{\mu}}, \overline{\hat{\theta}}) \text { with } \overline{\hat{\theta}}_{\hat{x}}:=\int_{0}^{1} \hat{\theta}_{\hat{x}, s} \mathrm{~d} \pi_{\hat{x}}(s), \overline{\hat{\theta}}=\left(\overline{\hat{\theta}}_{\hat{x}}\right)_{\hat{x} \in \omega} \otimes \pi^{\omega},
\end{gathered}
$$

where $\hat{\mu}, \hat{\theta}$ are the measures defined in (3), (4) and $\left(\pi_{\hat{x}}\right)_{\hat{x} \in \omega}$ is the familly of probability measures on $(0,1)$ stemming from the desintegration of the measure $\pi$ with respect to its projection $\pi^{\omega}$ on $\omega$ :

$$
\pi=\left(\pi_{\hat{x}}\right)_{\hat{x} \in \omega} \otimes \pi^{\omega}, \quad \pi^{\omega}=P_{\omega} \# \pi .
$$

Proposition 1. The images of $\nabla \mathcal{Y}_{3 \times 2,0}(\Omega)$ and $\nabla \mathcal{Y} \mathcal{V}_{3 \times 2,0}(\Omega)$ by the two maps $\Theta$ and $\Xi$ are exactly the two spaces $\nabla \mathcal{Y}_{3 \times 2}(\omega)$ and $\nabla \mathcal{Y} \mathcal{V}_{3 \times 2}(\omega)$.

Proof. a) Let us prove $\Theta\left(\nabla \mathcal{Y}_{3 \times 2,0}(\Omega)\right) \subset \nabla \mathcal{Y}_{3 \times 2}(\omega)$. Let $\nu=\Theta(\mu)$ with $\mu \in \nabla \mathcal{Y}_{3 \times 2,0}(\Omega)$. We must establish that $\nu$ satisfies the conditions (i), (ii) of Theorem 1 and (iii)' of Remark 1 . Since $\mu$ belongs to $\nabla \mathcal{Y}_{3 \times 2,0}(\Omega)$ there exists $u_{\varepsilon}$ in $W_{\Gamma_{0}}^{1, p}\left(\Omega, \mathbf{R}^{3}\right)$ such that (3) holds. Let $u$ be a cluster point of $\left(u_{\varepsilon}\right)_{\varepsilon>0}$ in $W_{\Gamma_{0}}^{1, p}\left(\Omega, \mathbf{R}^{3}\right)$. Classically, we have, for a.e. $x$ in $\Omega$,

$$
\nabla u(x)=\int_{\mathcal{M}^{3 \times 3}} \lambda \mathrm{d} \mu_{x}
$$

According to (3), we also have for a.e. $x$ in $\Omega$,

$$
\nabla u(x)=\left(\int_{\mathcal{M}^{3 \times 2}} \hat{\lambda} \mathrm{d} \hat{\mu}_{x}, 0\right)
$$


so that $u$ does not depends on the variable $x_{3}$, and $u \in W_{\gamma_{0}}^{1, p}\left(\omega, \mathbf{R}^{3}\right)$. Integrating $(5)$ over $(0,1)$ gives, for a.e. $\hat{x}$ in $\omega$

$$
\begin{aligned}
\hat{\nabla} u(\hat{x}) & =\int_{\mathcal{M}^{3 \times 2}} \hat{\lambda} \mathrm{d} \overline{\hat{\mu}}_{\hat{x}} \\
& =\int_{\mathcal{M}^{3 \times 2}} \hat{\lambda} \mathrm{d} \nu_{\hat{x}}
\end{aligned}
$$

which proves (i).

Let now $\psi: \mathcal{M}^{3 \times 2} \longrightarrow \mathbf{R}$ be a $W^{1, p}\left(\omega, \mathbf{R}^{3}\right)$-quasiconvex function satisfying $|\psi(\hat{\lambda})| \leq \beta\left(1+|\hat{\lambda}|^{p}\right)$ and set $\tilde{\psi}(\lambda):=\psi(\hat{\lambda})$ which defines a $W^{1, p}\left(\Omega, \mathbf{R}^{3}\right)$-quasiconvex function satisfying $|\tilde{\psi}(\lambda)| \leq \beta\left(1+|\lambda|^{p}\right)$. Since $\mu$ belongs to $\nabla \mathcal{Y}_{3 \times 3}(\Omega)$, (ii) holds and, for a.e. $x$ in $\Omega$,

$$
\begin{aligned}
\psi(\widehat{\nabla} u(\hat{x}))=\tilde{\psi}(\nabla u(x)) & \leq \int_{\mathcal{M}^{3 \times 3}} \tilde{\psi}(\lambda) \mathrm{d} \mu_{x} \\
& =\int_{\mathcal{M}^{3 \times 2}} \psi(\hat{\lambda}) \mathrm{d} \hat{\mu}_{x} .
\end{aligned}
$$

Integrating $(6)$ on $(0,1)$ gives, for a.e. $\hat{x}$ in $\omega$,

$$
\psi(\widehat{\nabla} u(\hat{x})) \leq \int_{\mathcal{M}^{3 \times 2}} \psi(\hat{\lambda}) \mathrm{d} \overline{\hat{\mu}}_{\hat{x}}
$$

which proves (ii). Assertion (iii') may be easily established and left to the reader.

b) We prove $\nabla \mathcal{Y}_{3 \times 2}(\omega) \subset \Theta\left(\nabla \mathcal{Y}_{3 \times 2,0}(\Omega)\right)$. Let $\nu \in \nabla \mathcal{Y}_{3 \times 2}(\omega)$ and $\left(v_{\varepsilon}\right)_{\varepsilon>0}$ a bounded sequence in $W_{\gamma_{0}}^{1, p}\left(\omega, \mathbf{R}^{3}\right)$ generating $\nu$. We set

$$
u_{\varepsilon}(x)=v_{\varepsilon}(\hat{x}) .
$$

Let us now consider the measures $\mu_{\varepsilon}=\left(\delta_{\nabla u_{\varepsilon}(x)}\right)_{x \in \Omega} \otimes \mathcal{L}$ and $\hat{\mu}_{\varepsilon}=\left(\delta_{\widehat{\nabla} u_{\varepsilon}(x)}\right)_{x \in \Omega} \otimes \mathcal{L}$ in respectively $\mathcal{Y}_{3 \times 3}(\Omega)$ and $\mathcal{Y}_{3 \times 2}(\Omega)$. It is easily seen that the sequences $\left(\mu_{\varepsilon}\right)_{\varepsilon>0}$ and $\left(\hat{\mu}_{\varepsilon}\right)_{\varepsilon>0}$ are tight so that, from Prokhorov's theorem (see Th. 5 in Appendix), there exit $\mu \in \nabla \mathcal{Y}_{3 \times 3}(\Omega)$ and $\hat{\mu} \in \mathcal{Y}_{3 \times 2}(\Omega)$ satisfying

$$
\begin{aligned}
& \mu_{\varepsilon} \stackrel{\text { nar }}{\longrightarrow} \mu, \\
& \hat{\mu}_{\varepsilon} \stackrel{\text { nar }}{\longrightarrow} \hat{\mu} .
\end{aligned}
$$

Since moreover $\frac{\partial u_{\varepsilon}}{\partial x_{3}}=0$, one easily deduce

$$
\mu_{x}=\hat{\mu}_{x} \otimes \delta_{0}
$$

Consequently $\mu$ belongs to $\nabla \mathcal{Y}_{3 \times 2,0}(\Omega)$.

It remains to establish that $\overline{\hat{\mu}}_{\hat{x}}=\nu_{\hat{x}}$. Since $\widehat{\nabla} u_{\varepsilon}=\nabla v_{\varepsilon}$ in $L^{p}\left(\Omega, \mathbf{R}^{3}\right)$, the sequences $\left(\widehat{\nabla} u_{\varepsilon}\right)_{\varepsilon>0}$ and $\left(\nabla v_{\varepsilon}\right)_{\varepsilon>0}$ generate the same Young measure in $\mathcal{Y}_{3 \times 2}(\Omega)$. As moreover $\left(\nabla u_{\varepsilon}\right)_{\varepsilon>0}$ generates $\nu \otimes \mathrm{d} x_{3}$, one has $\hat{\mu}=\nu \otimes \mathrm{d} x_{3}$ so that $\overline{\hat{\mu}}_{\hat{x}}=\nu_{\hat{x}}$.

c) We establish $\Xi\left(\nabla \mathcal{Y} \mathcal{V}_{3 \times 2,0}(\Omega)\right) \subset \nabla \mathcal{Y} \mathcal{V}_{3 \times 2}(\omega)$. Let $(\nu, m)=\Xi(\mu, \theta)$ with $\left.(\mu, \theta) \in \nabla \mathcal{Y} \mathcal{V}_{3 \times 2,0}(\Omega)\right)$. According to a) and b) above, it remains to establish iii) and iv).

Let $\psi$ be a $p$-homogeneous continuous function on $\mathcal{M}^{3 \times 2}$ satisfying $Q \psi(0)=0$ where $Q \psi$ denotes the quasiconvexification of $\psi$ on $\mathcal{M}^{3 \times 2}$ and set $\tilde{\psi}: \mathcal{M}^{3 \times 3} \rightarrow \mathbf{R}$ defined, for every $\lambda \in \mathcal{M}^{3 \times 3}$, by $\tilde{\psi}(\lambda)=\psi(\hat{\lambda})$. It is easily seen that $\tilde{\psi}$ is a $p$-homogeneous continuous function on $\mathcal{M}^{3 \times 3}$ and that $Q \tilde{\psi}(0)=0$ where $Q \tilde{\psi}$ now denotes the quasiconvexification of $\tilde{\psi}$ on $\mathcal{M}^{3 \times 3}$. 
Since $(\mu, \theta)$ is a $W_{\Gamma_{0}}^{1, p}\left(\Omega, \mathbf{R}^{3}\right)$-varifold, from iii) and iv) of Theorem 1, we deduce that for all Borel subset $B$ of $\omega$ :

$$
\begin{aligned}
\int_{\Omega} 1_{B}(\hat{x}) \int_{\mathcal{S}^{3 \times 3}} \tilde{\psi}(\lambda) \mathrm{d} \theta_{x}(\lambda) \mathrm{d} \pi_{s}(x) & +\int_{\Omega} 1_{B}(\hat{x})\left[\frac{\mathrm{d} \pi}{\mathrm{d} \mathcal{L}}(x) \int_{\mathcal{S}^{3 \times 3}} \tilde{\psi}(\lambda) \mathrm{d} \theta_{x}(\lambda)-\int_{\mathcal{M}^{3 \times 3}} \tilde{\psi}(\lambda) \mathrm{d} \mu_{x}(\lambda)\right] \mathrm{d} x \\
= & \int_{\Omega} 1_{B}(\hat{x})\left[\int_{\mathcal{S}^{3 \times 3}} \tilde{\psi}(\lambda) \mathrm{d} \theta_{x}(\lambda)\right] \mathrm{d} \pi(x)-\int_{\Omega} 1_{B}(\hat{x})\left[\int_{\mathcal{M}^{3 \times 3}} \tilde{\psi}(\lambda) \mathrm{d} \mu_{x}(\lambda)\right] \mathrm{d} x \geq 0 .
\end{aligned}
$$

Now, since $(\mu, \theta)$ belongs to $\nabla \mathcal{Y} \mathcal{V}_{3 \times 2,0}(\Omega), \theta_{x}=H \# \hat{\theta}_{x}$ and $\mu_{x}=\hat{\mu}_{x} \otimes \delta_{0}$. Moreover, taking into account that $\tilde{\psi}(\lambda)=\tilde{\psi}(\hat{\lambda}, 0)$, we obtain

$$
\int_{\Omega} 1_{B}(\hat{x})\left[\int_{\mathcal{S}^{3 \times 2}} \tilde{\psi}(\hat{\lambda}, 0) \mathrm{d} \hat{\theta}_{x}\right] \mathrm{d} \pi(x)-\int_{\Omega} 1_{B}(\hat{x})\left[\int_{\mathcal{M}^{3 \times 2}} \tilde{\psi}(\lambda) \mathrm{d} \hat{\mu}_{x}\right] \mathrm{d} x \geq 0 .
$$

Let us consider the slicing decomposition of $\pi: \pi=\left(\pi_{\hat{x}}\right)_{\hat{x} \in \omega} \otimes \pi^{\omega}$ where $\pi^{\omega}$ is the projection of $\pi$ on $\omega$. According to the slicing Theorem 4 (cf Appendix) applied to the first term of (7) and to Fubini's theorem for the second term, (7) becomes

$$
\int_{B}\left[\int_{0}^{1} \int_{\mathcal{S}^{3 \times 2}} \psi(\hat{\lambda}) \mathrm{d} \hat{\theta}_{\hat{x}, s} \mathrm{~d} \pi_{\hat{x}}(s)\right] \mathrm{d} \pi^{\omega}(\hat{x})-\int_{B}\left[\int_{0}^{1} \int_{\mathcal{M}^{3 \times 2}} \psi(\hat{\lambda}) \mathrm{d} \hat{\mu}_{\hat{x}, s} \mathrm{~d} x\right] \mathrm{d} \hat{x} \geq 0
$$

that is, with the definition of the probability measures $m_{\hat{x}}$ and $\nu_{\hat{x}}$,

$$
\int_{B}\left[\int_{\mathcal{S}^{3 \times 2}} \psi(\hat{\lambda}) \mathrm{d} m_{\hat{x}}\right] \mathrm{d} \pi^{\omega}(\hat{x})-\int_{B}\left[\int_{\mathcal{M}^{3 \times 2}} \psi(\hat{\lambda}) \mathrm{d} \nu_{\hat{x}}\right] \mathrm{d} \hat{x} \geq 0 .
$$

Collecting the regular and singular part in (8), according to the Lebesgue-Nikodym decomposition of the measure $\pi^{\omega}$

$$
\pi^{\omega}=\frac{\mathrm{d} \pi^{\omega}}{\mathrm{d} \hat{\mathcal{L}}} \hat{\mathcal{L}}+\pi_{s}^{\omega}
$$

we obtain

$$
\int_{B}\left[\int_{\mathcal{S}^{3 \times 2}} \psi \mathrm{d} m_{\hat{x}}\right] \mathrm{d} \pi_{s}^{\omega}(\hat{x})+\int_{B}\left[\frac{\mathrm{d} \pi^{\omega}}{\mathrm{d} \hat{\mathcal{L}}}(\hat{x}) \int_{\mathcal{S}^{3 \times 2}} \psi \mathrm{d} m_{\hat{x}}-\int_{\mathcal{M}^{3 \times 2}} \psi \mathrm{d} \nu_{\hat{x}}\right] \mathrm{d} \hat{x} \geq 0 .
$$

As the measures $\pi_{s}^{\omega}$ and $\hat{\mathcal{L}}$ are mutually singular, we deduce

$$
\begin{aligned}
& \frac{\mathrm{d} \pi^{\omega}}{\mathrm{d} \hat{\mathcal{L}}}(\hat{x}) \int_{\mathcal{S}^{3 \times 2}} \psi \mathrm{d} m_{\hat{x}}-\int_{\mathcal{M}^{3 \times 2}} \tilde{\psi} \mathrm{d} \nu_{\hat{x}} \geq 0 \hat{\mathcal{L}} \text { a.e. } x \in \omega, \\
& \int_{\mathcal{S}^{3 \times 2}} \psi \mathrm{d} m_{\hat{x}} \geq 0 \pi_{s}^{\omega} \text { a.e. } x \in \omega,
\end{aligned}
$$

which ends the proofs of (iii) and (iv).

d) We establish $\nabla \mathcal{Y} \mathcal{V}_{3 \times 2}(\omega) \subset \Xi\left(\nabla \mathcal{Y} \mathcal{V}_{3 \times 2,0}(\Omega)\right)$. Let $(\nu, m) \in \nabla \mathcal{Y} \mathcal{V}_{3 \times 2}(\omega)$ and $\left(v_{\varepsilon}\right)_{\varepsilon>0}$ generating $(\nu, m)$, that is to say:

$$
\begin{aligned}
& \left(\delta_{\nabla v_{\varepsilon}(\hat{x})}\right)_{\hat{x} \in \omega} \otimes \widehat{\mathcal{L}} \stackrel{\text { nar }}{\rightarrow} \nu \\
& \left(\delta_{\frac{\nabla v_{\varepsilon}}{\left|\nabla v_{\varepsilon}\right|}(\hat{x})}\right)_{\hat{x} \in \omega} \otimes\left|\nabla v_{\varepsilon}\right|^{p} \widehat{\mathcal{L}} \rightarrow m .
\end{aligned}
$$


Let us consider the function $u_{\varepsilon} \in W_{\Gamma_{0}}^{1, p}\left(\Omega, \mathbf{R}^{3}\right)$ defined by $u_{\varepsilon}(x)=v_{\varepsilon}(\hat{x})$ and the following measure of $\mathbf{M}^{+}(\Omega \times$ $\left.\mathcal{S}^{3 \times 3}\right)$ :

$$
\theta_{\varepsilon}=\left(\delta_{\frac{\nabla u_{\varepsilon}}{\left|\nabla u_{\varepsilon}\right|}(x)}\right)_{x \in \Omega} \otimes\left|\nabla u_{\varepsilon}\right|^{p} \mathcal{L}
$$

Since

$$
\sup _{\varepsilon>0} \theta_{\varepsilon}\left(\Omega \times \mathcal{S}^{3 \times 3}\right)=\int_{\Omega}\left|\nabla u_{\varepsilon}\right|^{p} \mathrm{~d} x<+\infty,
$$

there exists a subsequence of $\left(\theta_{\varepsilon}\right)_{\varepsilon>0}$ (non relabeled), weakly converging to some measure $\theta$ in $\mathbf{M}^{+}\left(\Omega \times \mathcal{S}^{3 \times 3}\right)$.

On the other hand, with the same arguments, one may easily establish that the measure

$$
\hat{\theta}_{\varepsilon}=\left(\delta_{\widehat{\nabla} u_{\varepsilon}}^{\left|\nabla \nabla u_{\varepsilon}\right|}(x)\right)_{x \in \Omega} \otimes\left|\widehat{\nabla} u_{\varepsilon}\right|^{p} \mathcal{L}
$$

weakly converges, for a non relabeled subsequence, to some measure $\hat{\theta}$ in $\mathbf{M}^{+}\left(\Omega \times \mathcal{S}^{3 \times 2}\right)$.

According to the definitions of the operator $\Xi$ and the space $\nabla \mathcal{Y} \mathcal{V}_{3 \times 2,0}(\Omega)$, it suffices to prove:

$$
(\mu, \theta) \in \nabla \mathcal{Y} \mathcal{V}_{3 \times 2,0}(\Omega)
$$

and

$$
m_{\hat{x}}=\int_{0}^{1} \hat{\theta}_{\hat{x}, s} \mathrm{~d} \pi_{\hat{x}}(s), \pi^{\omega} \text { a.e. } \hat{x} \in \omega .
$$

Proof of (9). To shorten notations, we do not distinguish a continuous function on $\mathcal{S}^{3 \times 3}$ or $\mathcal{S}^{3 \times 2}$ with its $p$ homogeneous extension. Taking into account b), it remains to establish $\pi:=P_{\Omega} \# \theta=P_{\Omega} \# \hat{\theta}$ and $\theta_{x}=H \# \hat{\theta}_{x}$ for $\pi$ a.e. $x \in \Omega$. A straightforward consequence of the definition of $u_{\varepsilon}$ is that $P_{\Omega} \# \theta$ and $P_{\Omega} \# \hat{\theta}$ are the weak limits of the measures $\left|\nabla u_{\varepsilon}\right|^{p} \mathcal{L}$ and $\left|\widehat{\nabla} u_{\varepsilon}\right|^{p} \mathcal{L}$. Let $\varphi \in \mathcal{C}_{0}(\Omega)$ and $\psi$ be a Lipschitz function on $\mathcal{S}^{3 \times 3}$. The weak convergence of $\theta_{\varepsilon}$ to $\theta$ in $\mathbf{M}\left(\Omega \times \mathcal{S}^{3 \times 3}\right)$ yields

$$
\lim _{\varepsilon \rightarrow 0} \int_{\Omega} \varphi(x) \psi\left(\nabla u_{\varepsilon}\right) \mathrm{d} x=\int_{\Omega} \varphi(x)\left(\int_{\mathcal{S}^{3 \times 3}} \psi(\lambda) \mathrm{d} \theta_{x}\right) \mathrm{d} \pi .
$$

On the other hand, since $\hat{\lambda} \mapsto \psi(\hat{\lambda}, 0)$ belongs to $\mathcal{C}\left(\mathcal{S}^{3 \times 2}\right)$, the weak convergence of $\hat{\theta}_{\varepsilon}$ to $\hat{\theta}$ in $\mathbf{M}\left(\Omega \times \mathcal{S}^{3 \times 2}\right)$ yields

$$
\lim _{\varepsilon \rightarrow 0} \int_{\Omega} \varphi(x) \psi\left(\widehat{\nabla} u_{\varepsilon}, 0\right) \mathrm{d} x=\int_{\Omega} \varphi(x)\left(\int_{\mathcal{S}^{3 \times 2}} \psi(\hat{\lambda}, 0) \mathrm{d} \hat{\theta}_{x}\right) \mathrm{d} \pi
$$

As

$$
\lim _{\varepsilon \rightarrow 0} \int_{\Omega} \varphi(x) \psi\left(\nabla u_{\varepsilon}\right) \mathrm{d} x=\lim _{\varepsilon \rightarrow 0} \int_{\Omega} \varphi(x) \psi\left(\widehat{\nabla} u_{\varepsilon}, 0\right) \mathrm{d} x
$$

(11) and (12) yield

$$
\int_{\mathcal{S}^{3 \times 3}} \psi(\lambda) \mathrm{d} \theta_{x}=\int_{\mathcal{S}^{3 \times 2}} \psi(\hat{\lambda}, 0) \mathrm{d} \hat{\theta}_{x} \pi \text { a.e } x \in \Omega,
$$

so that, according to the definition of $H \# \hat{\theta}_{x}$, and by a density argument, $\theta_{x}=H \# \hat{\theta}_{x}$ for $\pi$ a.e $x \in \Omega$.

Proof of (10). Let us recall that

$$
\pi=\left(\pi_{\hat{x}}\right)_{\hat{x} \in \omega} \otimes \pi^{\omega}, \quad \pi^{\omega}=P_{\omega} \# \pi
$$


and denote by $\hat{\pi}$ the weak limit of the measure $\left|\nabla v_{\varepsilon}\right|^{p} \hat{\mathcal{L}}$ in $\mathbf{M}(\omega)$. We claim that $\hat{\pi}=\pi^{\omega}$. Indeed, from the definition of $u_{\varepsilon}$, for every $\varphi \in \mathcal{C}_{0}(\omega)$, we have

$$
\begin{aligned}
\int_{\omega} \varphi(\hat{x}) \mathrm{d} \hat{\pi} & =\lim _{\varepsilon \rightarrow 0} \int_{\omega} \varphi(\hat{x})\left|\nabla v_{\varepsilon}\right|^{p} \mathrm{~d} \hat{x} \\
& =\lim _{\varepsilon \rightarrow 0} \int_{\Omega} \varphi(\hat{x})\left|\nabla u_{\varepsilon}\right|^{p} \mathrm{~d} x \\
& =\int_{\Omega} \varphi(\hat{x}) \mathrm{d} \pi=\int_{\omega} \varphi(\hat{x}) \mathrm{d} \pi^{\omega}
\end{aligned}
$$

where we have used $\pi^{\omega}=P_{\omega} \# \pi$ in the last equality.

Now, since $\left(\delta_{\frac{\nabla v_{\varepsilon}}{\left|\nabla v_{\varepsilon}\right|}(\hat{x})}\right)_{\hat{x} \in \omega} \otimes\left|\nabla v_{\varepsilon}\right|^{p} \widehat{\mathcal{L}} \rightarrow m=\left(m_{\hat{x}}\right)_{\hat{x} \in \omega} \otimes \hat{\pi}$ in $\mathbf{M}\left(\omega \times \mathcal{S}^{3 \times 2}\right)$, for every $\varphi \in \mathcal{C}_{0}\left(\omega \times \mathcal{S}^{3 \times 2}\right)$,

$$
\begin{aligned}
\lim _{\varepsilon \rightarrow 0} \int_{\omega} \varphi\left(\hat{x}, \nabla v_{\varepsilon}\right) \mathrm{d} \hat{x} & =\int_{\omega} \int_{\mathcal{S}^{3 \times 2}} \varphi(\hat{x}, \hat{\lambda}) \mathrm{d} m_{\hat{x}}(\hat{\lambda}) \mathrm{d} \hat{\pi}(\hat{x}) \\
& =\int_{\omega} \int_{\mathcal{S}^{3 \times 2}} \varphi(\hat{x}, \hat{\lambda}) \mathrm{d} m_{\hat{x}}(\hat{\lambda}) \mathrm{d} \pi^{\omega}(\hat{x}) .
\end{aligned}
$$

On the other hand

$$
\begin{aligned}
\lim _{\varepsilon \rightarrow 0} \int_{\omega} \varphi\left(\hat{x}, \nabla v_{\varepsilon}(\hat{x})\right) \mathrm{d} \hat{x} & =\lim _{\varepsilon \rightarrow 0} \int_{\Omega} \varphi\left(\hat{x}, \nabla v_{\varepsilon}(\hat{x})\right) \mathrm{d} x \\
& =\lim _{\varepsilon \rightarrow 0} \int_{\Omega} \varphi\left(\hat{x}, \hat{\nabla} u_{\varepsilon}(x)\right) \mathrm{d} x \\
& =\int_{\Omega \times \mathcal{S}^{3 \times 2}} \varphi(\hat{x}, \hat{\lambda}) \mathrm{d} \hat{\theta} \\
& =\int_{\Omega}\left(\int_{\mathcal{S}^{3 \times 2}} \varphi(\hat{x}, \hat{\lambda}) \mathrm{d} \hat{\theta}_{x}\right) \mathrm{d} \pi \\
& =\int_{\omega} \int_{0}^{1}\left(\int_{\mathcal{S}^{3 \times 2}} \varphi(\hat{x}, \hat{\lambda}) \mathrm{d} \hat{\theta}_{\hat{x}, s} \mathrm{~d} \pi_{\hat{x}}(s)\right) \mathrm{d} \pi^{\omega} .
\end{aligned}
$$

Collecting (13) and (14) and since $\varphi$ is arbitrary, we obtain (10).

\section{Young MEASURE FORMULATION}

According to the various notions introduced in Section 3, we now describe the Young measure formulation of the total energy associated with the layer.

\subsection{The Young measure formulation of the stored strain energy of the thin layer}

We introduce the Young measure formulation of the stored strain energy of the layer by defining the integral functional: $G_{\varepsilon}: \mathcal{Y}_{3 \times 3}(\Omega) \rightarrow \mathbf{R} \cup\{+\infty\}$,

$$
G_{\varepsilon}(\mu)=\left\{\begin{array}{l}
\int_{\Omega \times \mathcal{M}^{3 \times 3}} f\left(\hat{\lambda}, \frac{1}{\varepsilon} \lambda_{3}\right) \mathrm{d} \mu(x, \lambda) \text { if } \mu \in E \nabla \mathcal{Y}_{3 \times 3}(\Omega) \\
+\infty \text { otherwise. }
\end{array}\right.
$$


For each $\mu \in \nabla \mathcal{Y}_{3 \times 3}(\Omega)$, let us denote by $\mathbf{E} \mu$ its barycenter:

$$
\mathbf{E} \mu(x)=\int_{\mathcal{M}^{3 \times 3}} \lambda \mathrm{d} \mu_{x} .
$$

Clearly there exists a unique $u \in W_{\Gamma_{0}}^{1, p}\left(\Omega, \mathbf{R}^{3}\right)$, denoted by $\nabla^{-1} \mathbf{E} \mu$, such that $\nabla u(x)=\mathbf{E} \mu(x)$ for a.e. $x \in \Omega$. The exterior loading in the Young measure formulation is then given by: $L: \mathcal{Y}_{3 \times 3}(\Omega) \rightarrow \mathbf{R} \cup\{+\infty\}$,

$$
L(\mu)=\left\{\begin{array}{l}
\int_{\Omega} \tilde{g} \cdot \nabla^{-1} \mathbf{E} \mu \mathrm{d} x \text { if } \mu \in E \nabla \mathcal{Y}_{3 \times 3}(\Omega) \\
+\infty \text { otherwise. }
\end{array}\right.
$$

In order to shorten notations, we have adopted the same notaion $L$ to define the classical exterior loading and the exterior loading in the Young measure formulation. Note that if $\mu$ belongs to $E \mathcal{Y}_{3 \times 3}(\Omega)$ then $G_{\varepsilon}(\mu)-L(\mu)=$ $\tilde{F}_{\varepsilon}(u)-L(u)$ with $u=\nabla^{-1} \mathbf{E} \mu$.

The stored strain energy $G$ of the limit problem in the Young measure formulation is defined as follows: $G: \mathcal{Y}_{3 \times 2}(\omega) \rightarrow \mathbf{R} \cup\{+\infty\}$,

$$
G(\nu)=\left\{\begin{array}{l}
\int_{\omega \times \mathcal{M}^{3 \times 2}} f_{0}(\hat{\lambda}) \mathrm{d} \nu(\hat{x}, \hat{\lambda}) \text { if } \nu \in \nabla \mathcal{Y}_{3 \times 2}(\omega) \\
+\infty \text { otherwise. }
\end{array}\right.
$$

The limit exterior loading in the Young measure formulation is the following functional: $\bar{L}: \mathcal{Y}_{3 \times 2}(\omega) \rightarrow$ $\mathbf{R} \cup\{+\infty\}$

$$
\bar{L}(\nu)=\left\{\begin{array}{l}
\int_{\omega} \overline{\tilde{g}} \cdot \nabla^{-1} \mathbf{E}(\nu) \mathrm{d} \hat{x} \text { if } \nu \in \nabla \mathcal{Y}_{3 \times 2}(\omega) \\
+\infty \text { otherwise. }
\end{array}\right.
$$

\subsection{The $\boldsymbol{\Gamma}_{\boldsymbol{Y}}$-convergence in the Young measure formulation}

In this section, we are going to introduce a variational convergence, justifying the previous formulation of the limit energy. We begin by introducing a new weak notion of convergence between elements of $\mathcal{Y}_{3 \times 3}(\Omega)$ and $\mathcal{Y}_{3 \times 2}(\omega)$.

Definition 1. Let $\left(\mu_{\varepsilon}\right)_{\varepsilon>0}$ be a sequence in $\mathcal{Y}_{3 \times 3}(\Omega)$ and $\nu$ in $\mathcal{Y}_{3 \times 2}(\omega)$. We say that $\mu_{\varepsilon}$ weakly converges in the sense of membranes to $\nu$ and we write

$$
\mu_{\varepsilon} \stackrel{\text { mem }}{\rightarrow} \nu
$$

iff there exists $\mu$ in $\nabla \mathcal{Y}_{3 \times 2,0}(\Omega)$ such that $\mu_{\varepsilon} \stackrel{\text { nar }}{\longrightarrow} \mu$ and $\nu=\Theta(\mu)$.

It is worth noticing that, according to Proposition 1, the weak limit $\nu$ necessary belongs to $\nabla \mathcal{Y}_{3 \times 2}(\omega)$.

Let us now consider a sequence of functionals

$$
\begin{aligned}
& H_{\varepsilon}: \mathcal{Y}_{3 \times 3}(\Omega) \longrightarrow \mathbf{R} \cup\{+\infty\} \\
& H: \mathcal{Y}_{3 \times 2}(\omega) \longrightarrow \mathbf{R} \cup\{+\infty\}
\end{aligned}
$$

The following concept of convergence is very similar to that of $\Gamma$-convergence $($ see $[1,5])$. 


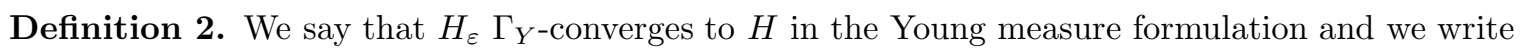

$$
H_{\varepsilon} \stackrel{\Gamma_{Y}}{\longrightarrow} H
$$

iff for all $\nu$ in $\mathcal{Y}_{3 \times 2}(\omega)$, both following assertions hold:

$$
\begin{aligned}
& \forall \mu_{\varepsilon} \in \mathcal{Y}_{3 \times 3}(\Omega), \text { s.t. } \mu_{\varepsilon} \stackrel{\text { mem }}{\longrightarrow} \nu, \quad H(\nu) \leq \liminf _{\varepsilon \rightarrow 0} H_{\varepsilon}\left(\mu_{\varepsilon}\right) \\
& \exists \nu_{\varepsilon} \in \mathcal{Y}_{3 \times 3}(\Omega) \text { s.t. } \nu_{\varepsilon} \stackrel{\text { mem }}{\longrightarrow} \nu, \quad H(\nu) \geq \underset{\varepsilon \rightarrow 0}{\limsup } H_{\varepsilon}\left(\nu_{\varepsilon}\right) .
\end{aligned}
$$

Let us consider the two following functionals $\Gamma_{Y} \liminf H_{\varepsilon}$ and $\Gamma_{Y} \lim \sup H_{\varepsilon}$ from $\mathcal{Y}_{3 \times 2}(\omega)$ into $\mathbf{R} \cup\{+\infty\}$, defined by

$$
\begin{aligned}
& \Gamma_{Y} \liminf H_{\varepsilon}(\nu)=\inf \left\{\liminf _{\varepsilon \rightarrow 0} H_{\varepsilon}\left(\mu_{\varepsilon}\right): \mu_{\varepsilon} \stackrel{\text { mem }}{\longrightarrow} \nu\right\} \\
& \Gamma_{Y} \limsup H_{\varepsilon}(\nu)=\inf \left\{\lim \sup _{\varepsilon \rightarrow 0} H_{\varepsilon}\left(\mu_{\varepsilon}\right): \mu_{\varepsilon} \stackrel{\text { mem }}{\rightarrow} \nu\right\} \text {. }
\end{aligned}
$$

It is easy to establish that these two functionals are lsc on $\mathcal{Y}_{3 \times 2}(\omega)$ and that $H_{\varepsilon} \stackrel{\Gamma_{Y}}{\longrightarrow} H$ iff one has, for every $\nu$ in $\mathcal{Y}_{3 \times 2}(\omega)$

$$
\Gamma_{Y} \lim \sup H_{\varepsilon}(\nu) \leq H(\nu) \leq \Gamma_{Y} \liminf H_{\varepsilon}(\nu) .
$$

In the proposition below, we establish that the $\Gamma_{Y}$-convergence is variational.

Proposition 2. Let us assume that $\left(H_{\varepsilon}\right)_{\varepsilon>0} \Gamma_{Y}$-converges to $H$ and let $\left(\mu_{\varepsilon}\right)_{\varepsilon>0}$ be a sequence of $\mathcal{Y}_{3 \times 3}(\Omega)$ satisfying

$$
H_{\varepsilon}\left(\mu_{\varepsilon}\right) \leq \inf \left\{H_{\varepsilon}(\mu): \mu \in \mathcal{Y}_{3 \times 3}(\Omega)\right\}+\varepsilon .
$$

Assume furthermore that $\left\{\mu_{\varepsilon}: \varepsilon>0\right\}$ is relatively compact for the weak convergence in the sense of membranes. Then any cluster point $\nu$ is a minimizer of $H$ in $\mathcal{Y}_{3 \times 2}(\omega)$ and

$$
\lim _{\varepsilon \rightarrow 0} \inf \left\{H_{\varepsilon}(\mu): \mu \in \mathcal{Y}_{3 \times 3}(\Omega)\right\}=H(\nu)
$$

Proof. Let $\nu \in \mathcal{Y}_{3 \times 2}(\omega)$ be such that $\mu_{\varepsilon} \stackrel{\text { mem }}{\rightarrow} \nu$. From the first assertion in Definition 2, we have

$$
H(\nu) \leq \liminf _{\varepsilon \rightarrow 0} H_{\varepsilon}\left(\mu_{\varepsilon}\right)
$$

Let $\mu$ be any element of $\mathcal{Y}_{3 \times 2}(\omega)$. From the second assertion in Definition 2 , there exists a sequence $\left(\nu_{\varepsilon}\right)_{\varepsilon>0}$ in $\mathcal{Y}_{3 \times 3}(\Omega)$ such that

$$
\limsup _{\varepsilon \rightarrow 0} H_{\varepsilon}\left(\nu_{\varepsilon}\right) \leq H(\mu)
$$

Inequalities (15) and (16) yield:

$$
\begin{aligned}
H(\nu) \leq \liminf _{\varepsilon \rightarrow 0} H_{\varepsilon}\left(\mu_{\varepsilon}\right) & =\liminf _{\varepsilon \rightarrow 0} \inf \left\{H_{\varepsilon}(\zeta): \zeta \in \mathcal{Y}_{3 \times 3}(\Omega)\right\} \\
& \leq \limsup _{\varepsilon \rightarrow 0} H_{\varepsilon}\left(\nu_{\varepsilon}\right) \leq H(\mu) .
\end{aligned}
$$

This shows that $\nu$ is a minimizer of $H$ in $\mathcal{Y}_{3 \times 2}(\omega)$. Taking $\mu=\nu$ in above inequalities gives $\lim _{\varepsilon \rightarrow 0} \inf \left\{H_{\varepsilon}(\zeta)\right.$ : $\left.\zeta \in \mathcal{Y}_{3 \times 3}(\Omega)\right\}=H(\nu)$. 


\subsection{The main result in the Young measure formulation}

Let us define the total energies in the Young measure formulation:

$$
H_{\varepsilon}:=G_{\varepsilon}-L, \quad H=G-\bar{L}
$$

Theorem below is our main result.

Theorem 2. Let us consider the sequence of integral functionals $\left(H_{\varepsilon}\right)_{\varepsilon>0}$ and $H$ defined above. Then we have

(i) Compactness: if $\sup _{\varepsilon>0} H_{\varepsilon}\left(\mu_{\varepsilon}\right)<+\infty$ then $\left\{\mu_{\varepsilon}: \varepsilon>0\right\}$ is relatively compact for the weak convergence in the sense of membrane.

(ii) Convergence: $H_{\varepsilon} \stackrel{\Gamma_{Y}}{\longrightarrow} H=G-\bar{L}$.

(iii) Let $\left(\mu_{\varepsilon}\right)_{\varepsilon>0}$ satisfying $H_{\varepsilon}\left(\mu_{\varepsilon}\right) \leq \varepsilon+\inf _{\mathcal{Y}_{3 \times 3}(\Omega)} H_{\varepsilon}$, and associated with some $u_{\varepsilon}$ in $W_{\Gamma_{0}}^{1, p}\left(\Omega, \mathbf{R}^{3}\right)$. Let moreover $\nu$ be a cluster point of $\left(\mu_{\varepsilon}\right)_{\varepsilon>0}$ for the weak convergence in the sense of membrane (see(i)), then $\nu$ is a minimizer of $\min _{\mathcal{Y}_{3 \times 2}(\omega)} H$ and $u_{\varepsilon}$ weakly converges to some $u$ in $W_{\Gamma_{0}}^{1, p}\left(\Omega, \mathbf{R}^{3}\right)$ which belongs to $W_{\gamma_{0}}^{1, p}\left(\omega, \mathbf{R}^{3}\right)$. For a.e. $\hat{x}$ in $\omega, \nabla u(\hat{x})$ is a barycenter of $\nu_{\hat{x}}$ and $u$ is a minimizer of the classical formulation

$$
\min \left\{F(u)-\bar{L}(u): u \in W_{\gamma_{0}}^{1, p}\left(\omega, \mathbf{R}^{3}\right)\right\}
$$

(iv) If $\nu$ is a minimizer of $\min _{\mathcal{Y}_{3 \times 2}(\omega)} H$ then the barycenter field

$$
\hat{x} \mapsto \int_{\mathcal{M}^{3 \times 2}} \hat{\lambda} \mathrm{d} \nu_{\hat{x}}
$$

is the gradient of a minimizer of the classical formulation

$$
\min \left\{F(u)-\bar{L}(u): u \in W_{\gamma_{0}}^{1, p}\left(\omega, \mathbf{R}^{3}\right)\right\}
$$

which then posseses an integral representation.

Proof.

Proof of (i). From the equiboundedness $\sup _{\varepsilon>0} H\left(\mu_{\varepsilon}\right)<+\infty$, we obviously obtain

$$
\left\{\begin{array}{l}
\mu_{\varepsilon}=\left(\delta_{\nabla u_{\varepsilon}(x)}\right)_{x \in \Omega} \otimes \mathcal{L}, \\
H_{\varepsilon}\left(\mu_{\varepsilon}\right)=\int_{\Omega} f\left(\widehat{\nabla} u_{\varepsilon}, \frac{1}{\varepsilon} \frac{\partial u_{\varepsilon}}{\partial x_{3}}\right) \mathrm{d} x-\int_{\Omega} \tilde{g} \cdot u_{\varepsilon} \mathrm{d} x, \\
\left(\nabla u_{\varepsilon}\right)_{\varepsilon>0} \text { bounded in } L^{p}\left(\Omega, \mathcal{M}^{3 \times 3}\right), \\
\left(\widehat{\nabla} u_{\varepsilon}\right)_{\varepsilon>0} \text { bounded in } L^{p}\left(\Omega, \mathcal{M}^{3 \times 2}\right), \\
\left(\frac{1}{\varepsilon} \frac{\partial u_{\varepsilon}}{\partial x_{3}}\right)_{\varepsilon>0} \text { bounded in } L^{p}\left(\Omega, \mathbf{R}^{3}\right) .
\end{array}\right.
$$

Since $\left(\nabla u_{\varepsilon}\right)_{\varepsilon>0}$ is bounded in $L^{p}\left(\Omega, \mathcal{M}^{3 \times 3}\right)$, the sequence $\left(\mu_{\varepsilon}\right)_{\varepsilon>0}$ is tight, and there exist $\mu \in \mathcal{Y}_{3 \times 3}(\Omega)$ and a non relabeled subsequence satisfying

$$
\mu_{\varepsilon} \stackrel{\text { nar }}{\rightarrow} \mu \text { in } \mathcal{Y}_{3 \times 3}(\Omega) .
$$


On the other hand, since $\left(\widehat{\nabla} u_{\varepsilon}\right)_{\varepsilon>0}$ is bounded in $L^{p}\left(\Omega, \mathcal{M}^{3 \times 2}\right)$, for the relabeled subsequence related to $\left(\mu_{\varepsilon}\right)_{\varepsilon>0}$, the sequence $\left(\hat{\mu}_{\varepsilon}\right)_{\varepsilon>0}, \hat{\mu}_{\varepsilon}=\left(\delta_{\widehat{\nabla} u_{\varepsilon}(x)}\right)_{x \in \Omega}$, is tight and there exists $\hat{\mu} \in \widehat{\nabla} \mathcal{Y}_{3 \times 2}(\Omega)$ and a non relabeled subsequence such that

$$
\hat{\mu}_{\varepsilon} \rightarrow \hat{\mu} \text { in } \mathcal{Y}_{3 \times 2}(\Omega) .
$$

Finally, since $\frac{\partial u_{\varepsilon}}{\partial x_{3}} \rightarrow 0$ in $\mathrm{E}^{p}\left(\Omega, \mathbf{R}^{3}\right)$, we classically have

$$
\left(\delta_{\frac{\partial u_{\varepsilon}}{\partial x_{3}}(x)}\right)_{x \in \Omega} \otimes \mathcal{L} \stackrel{\text { nar }}{\longrightarrow} \delta_{0} \otimes \mathcal{L} \text { in } \mathcal{Y}\left(\Omega, \mathbf{R}^{3}\right)
$$

One easily deduce that

$$
\mu_{x}=\hat{\mu}_{x} \otimes \delta_{0}
$$

so that $\mu$ belongs to $\nabla \mathcal{Y}_{3 \times 2,0}(\Omega)$. Let us set $\nu=\Theta(\mu)$, we have $\mu_{\varepsilon} \stackrel{\text { mem }}{\longrightarrow} \nu$.

Proof of (ii).

a) Let $\mu_{\varepsilon} \in \mathcal{Y}_{3 \times 3}(\Omega)$ and $\nu \in \mathcal{Y}_{3 \times 2}(\omega)$ be such that $\mu_{\varepsilon} \stackrel{\text { mem }}{\longrightarrow} \nu$. We establish

$$
H(\nu) \leq \liminf _{\varepsilon \rightarrow 0} H_{\varepsilon}\left(\mu_{\varepsilon}\right)
$$

Since $\mu_{\varepsilon} \stackrel{\text { mem }}{\longrightarrow} \nu$, there exists $\mu \in \nabla \mathcal{Y}_{3 \times 2,0}(\Omega)$ be such that $\mu_{\varepsilon} \stackrel{\text { nar }}{\longrightarrow} \mu$ and $\nu=\Theta(\mu)=\overline{\hat{\mu}}$. According to Proposition 1, $\nu$ belongs to $\nabla \mathcal{Y}_{3 \times 2}(\omega)$. On the other hand, one may assume $\sup _{\varepsilon>0} H_{\varepsilon}\left(\mu_{\varepsilon}\right)<+\infty$. Therefore

$$
\begin{aligned}
\liminf _{\varepsilon \rightarrow 0} H_{\varepsilon}\left(\mu_{\varepsilon}\right) & =\liminf _{\varepsilon \rightarrow 0}\left(\int_{\Omega \times \mathcal{M}^{3 \times 3}} f\left(\hat{\lambda}, \frac{1}{\varepsilon} \lambda_{3}\right) \mathrm{d} \mu_{\varepsilon}(x, \lambda)-\int_{\Omega} \tilde{g} \cdot u_{\varepsilon} \mathrm{d} x\right) \\
& \geq \liminf _{\varepsilon \rightarrow 0}\left(\int_{\Omega \times \mathcal{M}^{3 \times 3}} f_{0}(\hat{\lambda}) \mathrm{d} \mu_{\varepsilon}(x, \lambda)-\int_{\Omega} \tilde{g} \cdot u_{\varepsilon} \mathrm{d} x\right) \\
& \stackrel{\text { s.c.i }}{\geq} \int_{\Omega \times \mathcal{M}^{3 \times 3}} f_{0}(\hat{\lambda}) \mathrm{d} \mu(x, \lambda)-\int_{\Omega} \tilde{g} \cdot u \mathrm{~d} x \\
& =\int_{\Omega}\left(\int_{\mathcal{M}^{3 \times 3}} f_{0}(\hat{\lambda}) \mathrm{d} \hat{\mu}_{x} \otimes \delta_{0}\right) \mathrm{d} x-\int_{\Omega} \tilde{g} \cdot u \mathrm{~d} x \\
& \left.=\int_{\omega \times \mathcal{M}^{3 \times 2}} f_{0}(\hat{\lambda}) \mathrm{d} \overline{\mu_{x}} \mathrm{~d} \hat{x}-\int_{\omega} \overline{\tilde{g}} \cdot \nabla^{-1} \mathbf{E}(\overline{\hat{\mu}})\right) \mathrm{d} \hat{x} \\
& =H(\nu) .
\end{aligned}
$$

b) Let $\nu \in \mathcal{Y}_{3 \times 2}(\omega)$. We establish

$$
\Gamma_{Y} \limsup G_{\varepsilon}(\nu) \leq G(\nu) .
$$

To shorten notations and formulas, we do not take into acount the exterior loading which does not bring additional difficulties.

One may assume $G(\nu)<+\infty$. Therefore $\nu \in \nabla \mathcal{Y}_{3 \times 2}(\omega)$ and (see appendix),

$$
\begin{aligned}
& \exists v_{\eta} \in W_{\gamma_{0}}^{1, p}\left(\omega, \mathbf{R}^{3}\right) \text { such that } \nabla v_{\eta} \text { generates } \nu ; \\
& \left(\left|\nabla v_{\eta}\right|^{p}\right)_{\eta>0} \text { U.I. on } \omega
\end{aligned}
$$


hence

$$
G(\nu)=\lim _{\eta \rightarrow 0} \int_{\omega} f_{0}\left(\nabla v_{\eta}\right) \mathrm{d} \hat{x} .
$$

Let now $\xi$ be an arbitrary function in $\mathcal{D}\left(\omega, \mathbf{R}^{3}\right)$, and set

$$
\left\{\begin{array}{l}
u_{\varepsilon, \eta} \in W_{\Gamma_{0}}^{1, p}\left(\Omega, \mathbf{R}^{3}\right) \text { defined by } u_{\varepsilon, \eta}(x)=v_{\eta}(\hat{x})+\varepsilon x_{3} \xi(\hat{x}), \\
\mu_{\varepsilon, \eta}=\left(\delta_{\nabla u_{\varepsilon, \eta}(x)}\right)_{x \in \Omega} \otimes \mathcal{L} .
\end{array}\right.
$$

It is easy to prove that, when $\varepsilon \rightarrow 0$,

$$
\mu_{\varepsilon, \eta} \stackrel{\text { mem }}{\longrightarrow} \nu_{\eta}=\left(\delta_{\widehat{\nabla} v_{\eta}(\hat{x})}\right)_{\hat{x} \in \omega} \otimes \hat{\mathcal{L}}
$$

On the other hand

Consequently

$$
\lim _{\varepsilon \rightarrow 0} G_{\varepsilon}\left(\mu_{\varepsilon, \eta}\right)=\int_{\omega} f\left(\nabla v_{\eta}, \xi\right) \mathrm{d} \hat{x} .
$$

$$
\Gamma_{Y} \lim \sup G_{\varepsilon}\left(\nu_{\eta}\right) \leq \int_{\omega} f\left(\nabla v_{\eta}, \xi\right) \mathrm{d} \hat{x}
$$

Taking the infimum over $\xi$ in $\mathcal{D}\left(\omega, \mathbf{R}^{3}\right)$ in the right handside and using a classical localization argument, we then obtain

$$
\Gamma_{Y} \lim \sup G_{\varepsilon}\left(\nu_{\eta}\right) \leq \int_{\omega} f_{0}\left(\nabla v_{\eta}\right) \mathrm{d} \hat{x} .
$$

Since the functional $\Gamma_{Y} \lim \sup G_{\varepsilon}$ is lsc on $\mathcal{Y}_{3 \times 2}(\omega)$, letting $\eta \rightarrow 0$, (17) yields

$$
\Gamma_{Y} \limsup G_{\varepsilon}(\nu) \leq G(\nu) .
$$

c) According to the steps a) and b), we finally obtain, for every $\nu \in \mathcal{Y}_{3 \times 2}(\omega)$

$$
\Gamma_{Y} \lim \sup G_{\varepsilon}(\nu) \leq G(\nu) \leq \Gamma_{Y} \liminf G_{\varepsilon}(\nu)
$$

that is to say

and the proof of (ii) is complete.

$$
G_{\varepsilon} \stackrel{\Gamma_{Y}}{\longrightarrow} G
$$

Proof of (iii). The proof is a straightforward consequence of Proposition 2 and left to the reader.

Proof of (iv). Let $\nu$ be a minimizer of $\min \left\{H(\mu): \mu \in \mathcal{Y}_{3 \times 2}(\omega)\right\}$. Since $\nu$ belongs to $\nabla \mathcal{Y}_{3 \times 2}(\omega)$ and $Q f_{0}$ is quasiconvex

Therefore

$$
\left\{\begin{array}{l}
\exists u \in W_{\gamma_{0}}^{1, p}\left(\omega, \mathbf{R}^{3}\right), \hat{\nabla} u(\hat{x})=\int_{\mathcal{M}^{3 \times 2}} \hat{\lambda} \mathrm{d} \nu_{\hat{x}}(\hat{\lambda}) \quad \text { a.e. } \hat{x} \in \omega \\
Q f_{0}\left(\int_{\mathcal{M}^{3 \times 2}} \hat{\lambda} \mathrm{d} \nu_{\hat{x}}(\hat{\lambda})\right) \leq \int_{\mathcal{M}^{3 \times 2}} Q f_{0}(\hat{\lambda}) \mathrm{d} \nu_{\hat{x}}(\hat{\lambda}) .
\end{array}\right.
$$

$$
\begin{aligned}
\int_{\omega} Q f_{0}(\hat{\nabla} u(\hat{x})) \mathrm{d} \hat{x} & \leq \int_{\omega} \int_{\mathcal{M}^{3 \times 2}} Q f_{0}(\hat{\lambda}) \mathrm{d} \nu_{\hat{x}}(\hat{\lambda}) \mathrm{d} x \\
& \leq \int_{\omega} \int_{\mathcal{M}^{3 \times 2}} f_{0}(\hat{\lambda}) \mathrm{d} \nu_{\hat{x}}(\hat{\lambda}) \mathrm{d} x \\
& =G(\nu) .
\end{aligned}
$$


Consequently

$$
\begin{aligned}
F(u)-L(u) \leq H(\nu) & =\min \left\{H(\mu): \mu \in \mathcal{Y}_{3 \times 2}(\omega)\right\} \\
& =\min \left\{F(v)-L(v): v \in W_{\gamma_{0}}^{1, p}\left(\omega, \mathbf{R}^{3}\right)\right\}
\end{aligned}
$$

where the last equality is a consequence of a well known relaxation result (see [10], Th. 4.4 p. 67).

Remark 2. The previous result of variational convergence and the expression of $G(\nu)$ when $\nu$ belongs to $E \nabla \mathcal{Y}_{3 \times 2}(\omega)$ allow us to suggest a modeling of thin films of material with bulk energy $f$. It should be a membrane model with a surfacic strain energy density function $f_{0}$ so that the total strain energy involved by a displacement $u: \omega \rightarrow \mathbf{R}^{3}$, is $\int_{\omega} f_{0}(\nabla u(\hat{x})) \mathrm{d} \hat{x}$, where $f_{0}$ is not necessarily quasi-convex. If $f$ exhibits potential wells, it will be the same for $f_{0}$, thus this model may account for microstructures in thin films. A "variational" modeling of thin films was previously obtained in [3] from three-dimensional nonlinear elasticity but augmented with a classical term for interfacial surface strain. Neglecting formally the contribution of this term in their modeling provides a strain energy density function like our $f_{0}$. Thus all their considerations concerning microstructures are valuable for our modeling: a major fact is to predict the existence of exact untwinned austenite/martinsite interfaces.

\section{Oscillation-CONCENTRATION EFFECTS: THE VARIFOLD FORMULATION}

In this section the integral functional associated with the exterior loading, is that of previous section, and we assume that $\Gamma_{0}$ is the lateral boundary $\Gamma=\gamma \times(0,1)$ where $\gamma=\partial \omega$. In order to further take into account concentration effects for gradients minimizing sequences, according to the notion of varifold introduced in Section 3, we now describe the varifold formulation of the energy associated with the layer. For capturing possible gradient concentration on the boundary $\Gamma$ of $\Omega$, we extend $\Omega$ to the open bounded subset $\tilde{\Omega}=\tilde{\omega} \times(0,1)$ where $\tilde{\omega}$ is an arbitrary bounded open subset of $\mathbf{R}^{2}$ satisfying $\omega \subset \bar{\omega} \subset \tilde{\omega}$. We will denote by $\tilde{u}$ the extension by zero on $\tilde{\Omega} \backslash \Omega$ of any function $u \in W_{0}^{1, p}\left(\Omega, \mathbf{R}^{3}\right)$ and the same notation $\tilde{u}$ holds for the extension by zero on $\tilde{\omega} \backslash \omega$ of any functions $u \in W_{0}^{1, p}\left(\omega, \mathbf{R}^{3}\right)$. We will use the same notations $\mathcal{L}, \widehat{\mathcal{L}}$ to denote respectively the Lebesgue measure on $\Omega$ or $\tilde{\Omega}$ and $\omega$ or $\tilde{\omega}$.

The following spaces $\mathcal{Y} \mathcal{V}_{3 \times 3}(\tilde{\Omega}), E \nabla \mathcal{Y} \mathcal{V}_{3 \times 3}(\tilde{\Omega}), \nabla \mathcal{Y} \mathcal{V}_{3 \times 3}(\tilde{\Omega}), \nabla \mathcal{Y}_{3 \times 2,0}(\tilde{\Omega}), \mathcal{Y} \mathcal{V}_{3 \times 2}(\tilde{\omega})$ and $\nabla \mathcal{Y} \mathcal{V}_{3 \times 2}(\tilde{\omega})$ are respectively defined like the spaces $\mathcal{Y} \mathcal{V}_{3 \times 3}(\Omega), E \nabla \mathcal{Y} \mathcal{V}_{3 \times 3}(\Omega), \nabla \mathcal{Y V}_{3 \times 3}(\Omega), \nabla \mathcal{Y}_{3 \times 2,0}(\Omega), \mathcal{Y} \mathcal{V}_{3 \times 2}(\omega)$ and $\nabla \mathcal{Y} \mathcal{V}_{3 \times 2}(\omega)$ of Section 3, where $\tilde{\Omega}, \tilde{\omega}$ are substituted for $\Omega, \omega, \tilde{u}$ for $u$ and the generated functions $u_{\varepsilon}$ for $\tilde{u}_{\varepsilon}$.

The following lemma, whose proof is very easy, makes precise the relation between the spaces $\nabla \mathcal{Y} \mathcal{V}_{3 \times 2}(\tilde{\omega})$ and $\nabla \mathcal{Y} \mathcal{V}_{3 \times 2}(\omega)$.

Lemma 1. If $(\nu, m)$ belongs to $\nabla \mathcal{Y} \mathcal{V}_{3 \times 2}(\tilde{\omega})$, then

$$
\left\{\begin{array}{l}
\nu=\nu\left\lfloor\omega \times \mathcal{M}^{3 \times 2}+\delta_{0} \otimes \mathcal{L}\lfloor\tilde{\omega} \backslash \omega,\right. \\
m=m\left\lfloor\omega \times \mathcal{S}^{3 \times 2}+m\left\lfloor(\tilde{\omega} \backslash \omega) \times \mathcal{S}^{3 \times 2},\right.\right.
\end{array}\right.
$$

$\left(\nu\left\lfloor\omega \times \mathcal{M}^{3 \times 2}, m\left\lfloor\omega \times \mathcal{S}^{3 \times 2}\right)\right.\right.$ belongs to $\nabla \mathcal{Y} \mathcal{V}_{3 \times 2}(\omega)$ and $m\left\lfloor(\tilde{\omega} \backslash \omega) \times \mathcal{S}^{3 \times 2}\right.$ is concentrated on $\gamma \times \mathcal{S}^{3 \times 2}$.

Note that, since the support of the measure $m\left\lfloor(\tilde{\omega} \backslash \omega) \times \mathcal{S}^{3 \times 2}\right.$ is included in $\gamma \times \mathcal{S}^{3 \times 2}$, the decomposition $m\left\lfloor\omega \times \mathcal{S}^{3 \times 2}+m\left\lfloor(\tilde{\omega} \backslash \omega) \times \mathcal{S}^{3 \times 2}\right.\right.$ of $m$ is equal to $m\left\lfloor\omega \times \mathcal{S}^{3 \times 2}+m\left\lfloor\gamma \times \mathcal{S}^{3 \times 2}\right.\right.$, thus does not depend on the choice of the extension $\tilde{\omega}$ of $\omega$. We will see that the measure $\delta_{0} \otimes \mathcal{L}\lfloor\tilde{\omega} \backslash \omega$ does not play a role in the limit functional. 


\subsection{The varifold formulation of the stored strain energy of the thin layer}

We introduce the varifold formulation of the stored strain energy of the thin layer by defining the integral functional: $G_{\varepsilon}: \mathcal{Y} \mathcal{V}_{3 \times 3}(\tilde{\Omega}) \rightarrow \mathbf{R} \cup\{+\infty\}$,

$$
G_{\varepsilon}(\mu, \theta)=\left\{\begin{array}{l}
\int_{\Omega \times \mathcal{M}^{3 \times 3}} f\left(\hat{\lambda}, \frac{1}{\varepsilon} \lambda_{3}\right) \mathrm{d} \mu(x, \lambda) \text { if }(\mu, \theta) \in E \nabla \mathcal{Y} \mathcal{V}_{3 \times 3}(\tilde{\Omega}) \\
+\infty \text { otherwise. }
\end{array}\right.
$$

Obviously, $G_{\varepsilon}(\mu, \theta)=\tilde{F}_{\varepsilon}(u)$ when $(\mu, \theta)$ belongs to the domain of $G_{\varepsilon}$ and is generated by $u$.

We make the following additional hypotheses on the behavior of the density $f_{0}$ at infinity: there exists a $p$-homogeneous function $f_{0}^{\infty}$ satisfying:

$$
\lim _{|\hat{\lambda}| \rightarrow+\infty} \frac{f_{0}(\hat{\lambda})-f_{0}^{\infty}(\hat{\lambda})}{|\hat{\lambda}|^{p}}=0 .
$$

When $f_{0}$ depends on the plane variable $\hat{x}$, this limit is assumed to be uniform with respect to $\hat{x}$. Note that $f_{0}^{\infty}$ is uniquely defined by (18) and is nothing but the recession function of $f_{0}$ of degree $p$ defined by

$$
f_{0}^{\infty}(\hat{\lambda})=\lim _{t \rightarrow+\infty} \frac{f_{0}(t \hat{\lambda})}{t^{p}} .
$$

The stored strain energy $G$ of the limit problem in the varifold formulation is defined as follows: $G: \mathcal{Y} \mathcal{V}_{3 \times 2}(\tilde{\omega}) \rightarrow$ $\mathbf{R} \cup\{+\infty\}$,

$$
G(\nu, m)=\left\{\begin{aligned}
\int_{\omega \times \mathcal{M}^{3 \times 2}}\left(f_{0}-f_{0}^{\infty}\right)(\hat{\lambda}) \mathrm{d} \nu & +\int_{\omega \times \mathcal{S}^{3 \times 2}} f_{0}^{\infty}(\hat{\lambda}) \mathrm{d} m \\
& +\int_{\gamma \times \mathcal{S}^{3 \times 2}} f_{0}^{\infty}(\hat{\lambda}) \mathrm{d} m \text { if }(\nu, m) \in \nabla \mathcal{Y} \mathcal{V}_{3 \times 2}(\tilde{\omega}) \\
+\infty \text { otherwise. } &
\end{aligned}\right.
$$

\subsection{The $\Gamma_{Y V}$-convergence in the varifold formulation}

In this section, we are going to introduce a variational convergence, justifying the previous formulation of the limit energy. We begin by introducing a new weak notion of convergence between elements of $\mathcal{Y} \mathcal{V}_{3 \times 3}(\tilde{\Omega})$ and $\mathcal{Y} \mathcal{V}_{3 \times 2}(\tilde{\omega})$.

Definition 3. Let $\left(\mu_{\varepsilon}, \theta_{\varepsilon}\right)_{\varepsilon>0}$ be a sequence in $\mathcal{Y} \mathcal{V}_{3 \times 3}(\tilde{\Omega})$ and $(\nu, m)$ be a varifold in $\mathcal{Y} \mathcal{V}_{3 \times 2}(\tilde{\omega})$. We say that $\left(\mu_{\varepsilon}, \theta_{\varepsilon}\right)$ weakly converges in the sense of membranes to $(\nu, m)$ and we write

$$
\left(\mu_{\varepsilon}, \theta_{\varepsilon}\right) \stackrel{\text { mem }}{\longrightarrow}(\nu, m)
$$

iff there exists $(\mu, \theta)$ in $\nabla \mathcal{Y} \mathcal{V}_{3 \times 2,0}(\tilde{\Omega})$ such that $\left(\mu_{\varepsilon}, \theta_{\varepsilon}\right) \rightarrow(\mu, \theta)$ and $(\nu, m)=\Xi(\mu, \theta)$.

It is worth noticing that, according to Proposition 1 , the weak limit $(\nu, m)$ belongs to $\nabla \mathcal{Y} \mathcal{V}_{3 \times 2}(\tilde{\omega})$.

Let us now consider a sequence of functionals

$$
H_{\varepsilon}: \mathcal{Y} \mathcal{V}_{3 \times 3}(\tilde{\Omega}) \longrightarrow \mathbf{R} \cup\{+\infty\}
$$

and

$$
H: \mathcal{Y} \mathcal{V}_{3 \times 2}(\tilde{\omega}) \longrightarrow \mathbf{R} \cup\{+\infty\}
$$


Definition 4. We say that $\left(H_{\varepsilon}\right)_{\varepsilon>0} \Gamma$-converges in the varifold formulation to $H$, and we write

$$
H_{\varepsilon} \stackrel{\Gamma_{Y V}}{\longrightarrow} H
$$

iff for all $(\nu, m)$ in $\mathcal{Y} \mathcal{V}_{3 \times 2}(\tilde{\omega})$, both following assertions hold:

$$
\begin{aligned}
& \forall\left(\mu_{\varepsilon}, \theta_{\varepsilon}\right) \in \mathcal{Y} \mathcal{V}_{3 \times 3}(\tilde{\Omega}), \text { s.t. }\left(\mu_{\varepsilon}, \theta_{\varepsilon}\right) \stackrel{\text { mem }}{\longrightarrow}(\nu, m), \\
& H(\nu, m) \leq \liminf _{\varepsilon \rightarrow 0} H_{\varepsilon}\left(\mu_{\varepsilon}, \theta_{\varepsilon}\right) \\
& \exists\left(\mu_{\varepsilon}, \theta_{\varepsilon}\right) \in \mathcal{Y} \mathcal{V}_{3 \times 3}(\tilde{\Omega}) \text { s.t. }\left(\mu_{\varepsilon}, \theta_{\varepsilon}\right) \stackrel{\text { mem }}{\longrightarrow}(\nu, m) \text { and } \\
& H(\nu, m) \geq \limsup _{\varepsilon \rightarrow 0} H_{\varepsilon}\left(\mu_{\varepsilon}, \theta_{\varepsilon}\right) .
\end{aligned}
$$

Raisonning like in the proof of Proposition 2, it is easy to establish that this concept of convergence is variational. We left the reader to state the analogue of Proposition 2. It is moreover easily seen that the integral functional $L$ associated with the exterior loading is a continuous perturbation of stored strain energies $G_{\varepsilon}$ for this convergence.

\subsection{The main result in the varifold formulation}

Theorem below is our main result.

Theorem 3. Let us consider the sequence of integral functionals $\left(G_{\varepsilon}\right)_{\varepsilon>0}$ and $G$ defined above. Then we have

(i) Compactness: if $\sup _{\varepsilon>0} G_{\varepsilon}\left(\mu_{\varepsilon}, \theta_{\varepsilon}\right)-L\left(\mu_{\varepsilon}\right)<+\infty$ then $\left\{\left(\mu_{\varepsilon}, \theta_{\varepsilon}\right): \varepsilon>0\right\}$ is relatively compact for the weak convergence in the sense of membrane.

(ii) Convergence: $G_{\varepsilon}-L \stackrel{\Gamma_{Y V}}{\longrightarrow} G-\bar{L}$.

(iii) Let $\left(\mu_{\varepsilon}, \theta_{\varepsilon}\right)$ satisfying $G_{\varepsilon}\left(\mu_{\varepsilon}, \theta_{\varepsilon}\right)-L\left(\mu_{\varepsilon}\right) \leq \varepsilon+\inf _{\mathcal{Y} \mathcal{V}_{3 \times 3}(\Omega)}\left(G_{\varepsilon}-L\right)$, associated with $u_{\varepsilon}$ in $W_{0}^{1, p}\left(\Omega, \mathbf{R}^{3}\right)$. Let $(\nu, m)$ be a cluster point of $\left(\left(\mu_{\varepsilon}, \theta_{\varepsilon}\right)\right)_{\varepsilon>0}$ for the weak convergence in the sense of membrane (see $\left.(i)\right)$. Then $(\nu, m)$ is a minimizer of $\min _{\mathcal{Y} \mathcal{V}_{3 \times 2}(\tilde{\omega})}(G-\bar{L})$, $u_{\varepsilon}$ weakly converges to some $u$ in $W_{0}^{1, p}\left(\Omega, \mathbf{R}^{3}\right)$ which belongs to $W_{0}^{1, p}\left(\omega, \mathbf{R}^{3}\right)$; for a.e. $\hat{x}$ in $\omega, \nabla u(\hat{x})$ is a barycenter of $\nu_{\hat{x}}$ and $u$ is a minimizer of the classical formulation

$$
\min \left\{F(u)-\bar{L}(u): u \in W_{0}^{1, p}\left(\omega, \mathbf{R}^{3}\right)\right\}
$$

Moreover the measure $m=\left(m_{\hat{x}}\right)_{\hat{x} \in \omega \cup_{\gamma}} \otimes \pi^{\omega}$ captures concentrations of $\left.\left(\nabla u_{\varepsilon}\right)_{\varepsilon>0}\right)$ in the following sense: its projection $\pi^{\tilde{\omega}}$ on $\tilde{\omega}$ is the projection on $\tilde{\omega}$ of the weak limit in $\mathbf{M}(\tilde{\Omega})$ of the measure $\left|\nabla u_{\varepsilon}\right|^{p} \mathcal{L}$.

(iv) If $(\nu, m)$ is a minimizer of $\min _{\mathcal{Y}_{3 \times 2}(\tilde{\omega})}(G-\bar{L})$ then the barycenter field

$$
\hat{x} \mapsto \int_{\mathcal{M}^{3 \times 2}} \hat{\lambda} \mathrm{d} \nu_{\hat{x}}
$$

is the gradient of a minimizer of the classical formulation

$$
\min \left\{F(u)-\bar{L}(u): u \in W_{0}^{1, p}\left(\omega, \mathbf{R}^{3}\right)\right\}
$$

which then posseses an integral representation.

Proof.

Proof of (i). From the proof of (i) of Theorem 2, there exists a subsequence of $\left(\mu_{\varepsilon}\right)_{\varepsilon>0}$, non relabeled, and $\mu \in \nabla \mathcal{Y}_{3 \times 2,0}(\tilde{\Omega})$ such that $\mu_{\varepsilon} \stackrel{\text { nar }}{\longrightarrow} \mu$. 
On the other hand, clearly

$$
\theta_{\varepsilon}\left(\tilde{\Omega} \times \mathcal{S}^{3 \times 3}\right)=\int_{\Omega}\left|\nabla u_{\varepsilon}\right|^{p} \mathrm{~d} x<+\infty .
$$

Therefore, for a non relabeled subsequence of $\left(\theta_{\varepsilon}\right)_{\varepsilon>0}$, there exists a subsequence and $\theta \in \mathbf{M}^{+}\left(\tilde{\Omega} \times \mathcal{S}^{3 \times 3}\right)$ such that $\theta_{\varepsilon} \rightarrow \theta$. Arguying as in the proof d) of Proposition 1, we easily establish that $(\mu, \theta) \in \nabla \mathcal{Y} \mathcal{V}_{3 \times 2,0}(\tilde{\Omega})$. Let us set $(\nu, m)=\Xi(\mu, \theta)$. Clearly, for the non relabeled subsequence corresponding to that of $\left(\theta_{\varepsilon}\right)_{\varepsilon>0}$, we have $\left(\mu_{\varepsilon}, \theta_{\varepsilon}\right) \stackrel{\text { mem }}{\longrightarrow}(\nu, \theta)$.

Proof of (ii). To shorten notations, we only work with the stored energies.

a) Let $\left(\mu_{\varepsilon}, \theta_{\varepsilon}\right) \in \mathcal{Y} \mathcal{V}_{3 \times 3}(\tilde{\Omega})$ and $(\nu, m) \in \mathcal{Y}_{3 \times 2}(\tilde{\omega})$ be such that $\left(\mu_{\varepsilon}, \theta_{\varepsilon}\right) \stackrel{\text { mem }}{\longrightarrow}(\nu, m)$. We establish

$$
G(\nu, m) \leq \liminf _{\varepsilon \rightarrow 0} G_{\varepsilon}\left(\mu_{\varepsilon}, \theta_{\varepsilon}\right)
$$

Since $\left(\mu_{\varepsilon}, \theta_{\varepsilon}\right) \stackrel{\text { mem }}{\longrightarrow}(\nu, m)$, there exists $(\mu, \theta)$ in $\nabla \mathcal{Y} \mathcal{V}_{3 \times 2,0}(\tilde{\Omega})$ such that

$$
\left(\mu_{\varepsilon}, \theta_{\varepsilon}\right) \rightarrow(\mu, \theta) \text { and }(\nu, m)=\Xi(\mu, m) .
$$

According to Proposition 1, $(\nu, m)$ belongs to $\nabla \mathcal{Y} \mathcal{V}_{3 \times 2}(\tilde{\omega})$. On the other hand, one may assume $\sup _{\varepsilon>0} G_{\varepsilon}\left(\mu_{\varepsilon}, \theta_{\varepsilon}\right)<$ $+\infty$. Therefore there exits some $u_{\varepsilon}$ in $W_{0}^{1, p}\left(\Omega, \mathbf{R}^{3}\right)$ such that $\tilde{u}_{\varepsilon}$ generates the varifold $(\mu, \theta) \in \nabla \mathcal{Y} \mathcal{V}_{3 \times 2,0}(\tilde{\Omega})$. More precisely

$$
\left\{\begin{array}{l}
\left(\delta_{\nabla \tilde{u}_{\varepsilon}(x)}\right)_{x \in \tilde{\Omega}} \otimes \mathcal{L} \stackrel{\text { nar }}{\longrightarrow} \mu, \\
\left(\delta_{\hat{\nabla} \tilde{u}_{\varepsilon}(x)}\right)_{x \in \tilde{\Omega}} \otimes \mathcal{L} \stackrel{\text { nar }}{\longrightarrow} \hat{\mu}, \\
\mu_{x}=\hat{\mu}_{x} \otimes \delta_{0} \\
\theta_{\varepsilon}:=\left(\delta_{\frac{\nabla \tilde{u}_{\varepsilon}}{\left|\nabla \tilde{u}_{\varepsilon}\right|}(x)}\right)_{x \in \tilde{\Omega}} \otimes\left|\nabla u_{\varepsilon}\right|^{p} \mathcal{L} \rightarrow \theta \\
\hat{\theta}_{\varepsilon}:=\left(\delta_{\frac{\hat{\nabla} \tilde{\varepsilon}_{\varepsilon}}{\left|\nabla \tilde{u}_{\varepsilon}\right|}(x)}\right)_{x \in \tilde{\Omega}} \otimes\left|\hat{\nabla} u_{\varepsilon}\right|^{p} \mathcal{L} \rightarrow \hat{\theta} \\
P_{\tilde{\Omega}} \# \theta=P_{\tilde{\Omega}} \# \hat{\theta}:=\pi, \\
\theta_{x}=H \# \hat{\theta}_{x} \pi \text { a.e. } x \in \tilde{\Omega} .
\end{array}\right.
$$

Noticing that $f_{0}^{\infty}(0)=0$, we have

$$
\begin{aligned}
\liminf _{\varepsilon \rightarrow 0} G_{\varepsilon}\left(\mu_{\varepsilon}, \theta_{\varepsilon}\right) & =\liminf _{\varepsilon \rightarrow 0} \int_{\Omega \times \mathcal{M}^{3 \times 3}} f\left(\hat{\lambda}, \frac{1}{\varepsilon} \lambda_{3}\right) \mathrm{d} \mu_{\varepsilon}(x, \lambda) \\
& \geq \liminf _{\varepsilon \rightarrow 0} \int_{\Omega \times \mathcal{M}^{3 \times 3}} f_{0}(\hat{\lambda}) \mathrm{d} \mu_{\varepsilon}(x, \lambda) \\
& =\liminf _{\varepsilon \rightarrow 0} \int_{\Omega} f_{0}\left(\widehat{\nabla} u_{\varepsilon}\right) \mathrm{d} x \\
& =\liminf _{\varepsilon \rightarrow 0}\left(\int_{\Omega}\left(f_{0}-f_{0}^{\infty}\right)\left(\widehat{\nabla} u_{\varepsilon}\right) \mathrm{d} x+\int_{\tilde{\Omega}} f_{0}^{\infty}\left(\widehat{\nabla} \tilde{u}_{\varepsilon}\right) \mathrm{d} x\right) \\
& \geq \liminf _{\varepsilon \rightarrow 0} \int_{\Omega}\left(f_{0}-f_{0}^{\infty}\right)\left(\widehat{\nabla} u_{\varepsilon}\right) \mathrm{d} x+\liminf _{\varepsilon \rightarrow 0} \int_{\tilde{\Omega}} f_{0}^{\infty}\left(\frac{\hat{\nabla} \tilde{u}_{\varepsilon}}{\left|\widehat{\nabla} \tilde{u}_{\varepsilon}\right|}\right)\left|\widehat{\nabla} \tilde{u}_{\varepsilon}\right|^{p} \mathrm{~d} x \\
& \stackrel{\text { s.c.i }}{\geq} \int_{\Omega \times \mathcal{M}^{3 \times 3}}\left(f_{0}-f_{0}^{\infty}\right)(\hat{\lambda}) \mathrm{d} \mu+\int_{\bar{\Omega} \times \mathcal{S}^{3 \times 2}} f_{0}^{\infty}(\hat{\lambda}) \mathrm{d} \hat{\theta} \\
& =\int_{\Omega}\left(\int_{\mathcal{M}^{3 \times 2}}\left(f_{0}-f_{0}^{\infty}\right)(\hat{\lambda}) \mathrm{d} \hat{\mu}_{x}\right) \mathrm{d} x+\int_{\bar{\Omega}}\left(\int_{\mathcal{S}^{3 \times 2}} f_{0}^{\infty}(\hat{\lambda}) \mathrm{d} \hat{\theta}_{x}\right) \mathrm{d} \pi .
\end{aligned}
$$


Where we have use the lower semicontinuity result (see Appendix, Prop. 3), the slicing theorem (see Appendix, Th. 4) and Lemma 1 in the last equality. By using Fubini's theorem in the first term and the slicing decomposition $\pi=\left(\pi_{\hat{x}}\right)_{\hat{x} \in \tilde{\omega}} \otimes \pi^{\tilde{\omega}}$ in the second term of $(19)$, since $(\nu, m)=\Xi(\mu, \theta)$, we finally obtain

$$
\begin{aligned}
\liminf _{\varepsilon \rightarrow 0} G_{\varepsilon}\left(\mu_{\varepsilon}, \theta_{\varepsilon}\right) & \geq \int_{\omega \times \mathcal{M}^{3 \times 2}}\left(f_{0}-f_{0}^{\infty}\right)(\hat{\lambda}) \mathrm{d} \nu+\int_{\bar{\omega} \times \mathcal{S}^{3 \times 2}} f_{0}^{\infty}(\hat{\lambda}) \mathrm{d} m \\
& =G(\nu, m) .
\end{aligned}
$$

b) We establish the upper bound in the definition of the $\Gamma_{Y V}$-convergence. One may assume $G(\nu, m)<+\infty$. Let $(\nu, m) \in \mathcal{Y} \mathcal{V}_{3 \times 2}(\tilde{\omega})$ and $\left(v_{n}\right)_{n \in \mathbf{N}}, v_{n} \in W_{0}^{1, p}\left(\omega, \mathbf{R}^{3}\right)$ generating the varifold $(\nu, m)$ :

$$
\begin{aligned}
& \nu_{n}=\left(\delta_{\nabla \tilde{v}_{n}(\hat{x})}\right)_{\hat{x} \in \tilde{\omega}} \otimes \hat{\mathcal{L}} \stackrel{\text { nar }}{\rightarrow} \nu \\
& m_{n}:=\left(\delta_{\frac{\nabla \tilde{v}_{n}}{\left|\nabla \tilde{v}_{n}\right|}(\hat{x})}\right)_{\hat{x} \in \tilde{\omega}} \otimes\left|\nabla \tilde{v}_{n}\right|^{p} \hat{\mathcal{L}} \rightarrow m
\end{aligned}
$$

with $\hat{\pi}_{n}=\left|\nabla \tilde{v}_{n}\right|^{p} \hat{\mathcal{L}} \rightarrow \pi^{\tilde{\omega}}=P_{\tilde{\omega}} \# m$. According to (18), it is easily seen that $\hat{x} \mapsto 1_{\omega}(\hat{x})\left(f_{0}-f_{0}^{\infty}\right)\left(\nabla \tilde{v}_{n}(\hat{x})\right)_{n \in \mathbf{N}}$ is uniformly integrable so that, according to Proposition 4 of the Appendix

$$
\int_{\omega \times \mathcal{M}^{3 \times 2}}\left(f_{0}-f_{0}^{\infty}\right)(\hat{\lambda}) \mathrm{d} \nu=\lim _{n \rightarrow+\infty} \int_{\omega}\left(f_{0}-f_{0}^{\infty}\right)\left(\nabla v_{n}\right) \mathrm{d} \hat{x} .
$$

On the other hand, since the measure $m_{n}$ weakly converges to the measure $m$ in $M\left(\tilde{\omega} \times \mathcal{S}^{3 \times 2}\right)$, from the continuity of $\hat{\lambda} \mapsto f_{0}^{\infty}(\hat{\lambda})$, we deduce that the measure $f_{0}^{\infty} m_{n}$ weakly converges to the measure $f_{0}^{\infty} m$ in $M\left(\tilde{\omega} \times \mathcal{S}^{3 \times 2}\right)$. Now, since $\bar{\omega} \times \mathcal{S}^{3 \times 2}$ is a compact subset of $\tilde{\omega} \times \mathcal{S}^{3 \times 2}$, Alexandrov's theorem yields

$$
\begin{aligned}
\int_{\bar{\omega} \times \mathcal{S}^{3 \times 2}} f_{0}^{\infty}(\hat{\lambda}) \mathrm{d} m & \geq \limsup _{n \rightarrow+\infty} \int_{\bar{\omega}} f_{0}^{\infty}\left(\nabla \tilde{v}_{n}\right) \mathrm{d} \hat{x} \\
& =\limsup _{n \rightarrow+\infty} \int_{\omega} f_{0}^{\infty}\left(\nabla v_{n}\right) \mathrm{d} \hat{x} .
\end{aligned}
$$

Combining (20) and (21) gives

$$
G(\nu, m) \geq \limsup _{n \rightarrow+\infty} \int_{\omega} f_{0}\left(\nabla v_{n}\right) \mathrm{d} \hat{x} .
$$

We end the proof as that of Theorem 2ii, b).

Remark 3. Obviously, our study is a first approach in modelling concentration effects in membranes. Actually, in order to obtain gradients concentrations, for instance on the boundary of the membrane, it would be necessary to deal with exterior loadings varying with $\varepsilon$ and concentrated near the boundary. This case is more involved because the associated integral functional $L_{\varepsilon}$ may be a noncontinuous perturbation of $G_{\varepsilon}$ for the $\Gamma_{Y V^{-} \text {-convergence. }}$

\section{Appendix}

For a general exposition of the theory of Young measures, we refer the reader to Balder [2], Valadier [12,13] and the references therein.

In all the Appendix, $\Omega$ is an open bounded subset of $\mathbf{R}^{N}$ and $E=\mathbf{R}^{d}, d=m \times N$ so that $\mathbf{R}^{d}$ is canonically isomorphic to the space $\mathbf{M}^{m \times N}$ of $m \times N$ matrices. 
Definition 5. We call Young measure on $\Omega \times E$, any positive measure $\mu \in \mathbf{M}^{+}(\Omega \times E)$ such that its image by the projection $\pi_{\Omega}$ on $\Omega$ is the Lebesgue measure $\mathcal{L}$ on $\Omega$ : for every Borel subset $B$ of $\Omega$

$$
\pi_{\Omega} \# \mu(B):=\mu(B \times E)=\mathcal{L}(B)
$$

We denote by $\mathcal{Y}(\Omega ; E)$ the set of all Young measures on $\Omega \times E$.

We equip $\mathcal{Y}(\Omega ; E)$ with the narrow topology, that is the weakest topology which makes the maps

$$
\mu \mapsto \int_{\Omega \times E} \varphi \mathrm{d} \mu
$$

continuous, where $\varphi$ runs through $\mathcal{C}_{b}(\Omega ; E)$. This topology induces the narrow convergence of Young measures defined as follows: let $\left(\mu_{n}\right)_{n \in \mathbf{N}}$ be a sequence of measures in $\mathcal{Y}(\Omega ; E)$ and $\mu \in \mathcal{Y}(\Omega ; E)$, then

$$
\mu_{n} \stackrel{\text { nar }}{\longrightarrow} \mu \Longleftrightarrow\left\{\begin{array}{l}
\forall \varphi \in \mathcal{C}_{b}(\Omega ; E) \\
\lim _{n \rightarrow+\infty} \int_{\Omega \times E} \varphi(x, \lambda) \mathrm{d} \mu_{n}(x, \lambda)=\int_{\Omega \times E} \varphi(x, \lambda) \mathrm{d} \mu(x, \lambda)
\end{array}\right.
$$

The following slicing property, is a generalization of Fubini's Theorem.

Theorem 4. Let $\mu$ be any Young measure in $\mathcal{Y}(\Omega ; E)$. There exists a family of probability measure $\left(\mu_{x}\right)_{x \in \Omega}$ on $E$, unique up to equality $\mathcal{L}$-a.e. such that

(i) $x \mapsto \int_{E} \psi(x, \Lambda) \mathrm{d} \mu_{x}$ is $\mathcal{L}$-measurable;

(ii) $\int_{\Omega \times E} \psi(x, \Lambda) \mathrm{d} \mu(x, \Lambda)=\int_{\Omega}\left(\int_{E} \psi(x, \Lambda) \mathrm{d} \mu_{x}(\Lambda)\right) \mathrm{d} x$

for each $\mu$-integrable function $\psi$. The familly $\left(\mu_{x}\right)_{x \in \Omega}$ is called a disintegration of the Young measure $\mu$ and we write $\mu=\left(\mu_{x}\right)_{x \in \Omega} \otimes \mathcal{L}$.

Let us define the tightness notion for Young measures.

Definition 6. A subset $\mathcal{H}$ of $\mathcal{Y}(\Omega ; E)$ is said to be tight if

$$
\forall \varepsilon>0, \exists \mathcal{K}_{\varepsilon} \text {, compact subset of } E \text {, such that } \sup _{\mu \in \mathcal{H}} \mu\left(\Omega \times E \backslash \mathcal{K}_{\varepsilon}\right)<\varepsilon
$$

Theorem below may be considered as the parametrized version of the classical Prokhorov compactness theorem.

Theorem 5 (Prokhorov's Compactness Theorem). Let $\left(\mu^{n}\right)_{n \in \mathbf{N}}$ be a tight sequence in $\mathcal{Y}(\Omega ; E)$. Then, there exists a subsequence $\left(\mu^{n_{k}}\right)_{k \in \mathbf{N}}$ of $\left(\mu^{n}\right)_{n \in \mathbf{N}}$ and $\mu$ in $\mathcal{Y}(\Omega ; E)$ such that

$$
\mu_{n_{k}} \stackrel{\text { nar }}{\rightarrow} \mu \text { in } \mathcal{Y}(\Omega ; E)
$$

Let $u: \Omega \rightarrow E$ be a given function and let us consider the image measure $\mu=G \# \mathcal{L}$ of $\mathcal{L}$ by the graph function $G$ of $u$ :

$$
G: \Omega \rightarrow \Omega \times E, x \mapsto(x, u(x))
$$


Since the image of $\mu$ by the projection on $\Omega$ is $\mathcal{L}, \mu$ belongs to $\mathcal{Y}(\Omega ; E)$. This measure, concentrated on the graph of $u$, is called the Young measure associated with the function $u$. By definition of the image measure, $\mu$ "acts" on $\mathcal{C}_{b}(\Omega ; E)$ as follows:

$$
\int_{\Omega \times E} \varphi(x, \lambda) \mathrm{d} \mu(x, \lambda)=\int_{\Omega} \varphi(x, u(x)) \mathrm{d} x \forall \varphi \in \mathcal{C}_{b}(\Omega ; E) .
$$

This shows that the probability familly $\left(\mu_{x}\right)_{x \in \Omega}$ associated to $\mu$ is $\left(\delta_{u(x)}\right)_{x \in \Omega}$.

Let now $\left(u_{n}\right)_{n \in \mathbf{N}}$ be a sequence of functions $u_{n}: \Omega \rightarrow E$ and consider the sequence of their associated Young measures $\left(\mu_{n}\right)_{n \in \mathbf{N}}, \mu_{n}=\left(\delta_{u_{n}(x)}\right)_{x \in \Omega} \otimes \mathcal{L}$. If $\mu_{n} \stackrel{\text { nar }}{\rightarrow} \mu$ in $\mathcal{Y}(\Omega ; E)$, the Young measure $\mu$ is said to be generated by the sequence of functions $\left(u_{n}\right)_{n \in \mathbf{N}}$. In general, $\mu$ is not associated with a function.

Here is a semicontinuity result related to non negative functions:

Proposition 3. Let $\varphi: \Omega \times E \rightarrow[0,+\infty]$ be a $\mathcal{B}(\Omega) \otimes \mathcal{B}(E)$ measurable function such that $\lambda \mapsto \varphi(x, \lambda)$ is lsc for a.e. $x$ in $\Omega$. Let moreover $\left(\mu_{n}\right)_{n \in \mathbf{N}}$ be a sequence of Young measures in $\mathcal{Y}(\Omega ; E)$ narrowly converging to some Young measure $\mu$ in $\mathcal{Y}(\Omega ; E)$. Then

$$
\int_{\Omega \times E} \varphi(x, \lambda) \mathrm{d} \mu(x, \lambda) \leq \liminf _{n \rightarrow+\infty} \int_{\Omega \times E} \varphi(x, \lambda) \mathrm{d} \mu_{n}(x, \lambda) .
$$

Let us recall the notion of uniform integrability: a sequence $\left(f_{n}\right)_{n \in \mathbf{N}}, f_{n}: \Omega \rightarrow \mathbf{R}$ is said to be uniformly integrable if

$$
\lim _{R \rightarrow+\infty} \sup _{n \in \mathbf{N}} \int_{\left[\left|f_{n}\right|>R\right]}\left|f_{n}\right|=0 .
$$

One may extend the set $\mathcal{C}_{b}\left(\Omega, \mathbf{R}^{m}\right)$ of test functions related to the narrow convergence as follows:

Proposition 4. Let $\left(\mu_{n}\right)_{n \in \mathbf{N}}$ be a sequence of Young measures associated with a sequence of functions $\left(u_{n}\right)_{n \in \mathbf{N}}$, narrowly converging to some Young measure $\mu$. On the other hand let $\varphi: \Omega \times E \rightarrow \mathbf{R}$ be a $\mathcal{B}(\Omega) \otimes \mathcal{B}(E)$ measurable function such that $\lambda \mapsto \varphi(x, \lambda)$ is continuous for a.e. $x$ in $\Omega$. Assume moreover that $x \mapsto \varphi\left(x, u_{n}(x)\right)$ is uniformly integrable. Then

$$
\int_{\Omega \times E} \varphi(x, \lambda) \mathrm{d} \mu(x, \lambda)=\lim _{n \rightarrow+\infty} \int_{\Omega \times E} \varphi\left(x, u_{n}(x)\right) \mathrm{d} x .
$$

In order to apply Proposition 4, the following result is fundamental.

Proposition 5. Let $\left(u_{n}\right)_{n \in \mathbf{N}}$ be a bounded sequence in $W_{\Gamma_{0}}^{1, p}\left(\Omega, \mathbf{R}^{m}\right)$ whose gradients generate a $W^{1, p}-Y o u n g$ measure $\mu$. Then there exists another sequence $\left(v_{n}\right)_{n \in \mathbf{N}}$ in $W_{\Gamma_{0}}^{1, p}\left(\Omega, \mathbf{R}^{m}\right)$, whose gradients generate the same Young measure $\mu$, and such that $\left(\left|\nabla v_{n}\right|^{p}\right)_{n \in \mathbf{N}}$ is uniformly integrable.

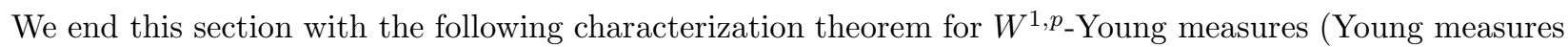
generated by gradients of $W^{1, p}$-functions), established by D. Kinderlehrer and P. Pedregal (see $\left.[8,10,11]\right)$.

Theorem 6. Let $p>1$. Then $\mu \in \mathcal{Y}(\Omega ; E)$ is a $W^{1, p}$-Young measure iff there exists $u \in W^{1, p}\left(\Omega, \mathbf{R}^{m}\right)$ such that the three following assertions hold:
i) $\nabla u(x)=\int_{E} \lambda \mathrm{d} \mu_{x}(\lambda)$ has
iii) $\int_{\Omega \times E}|\lambda|^{p} \mathrm{~d} \mu(x, \lambda)<+\infty$.

$$
\phi(\nabla u(x)) \leq \int_{E} \phi(\lambda) \mathrm{d} \mu_{x}(\lambda), \quad \text { a.a.x } \in \Omega
$$

ii) for all quasiconvex function $\phi$ satisfying $0 \leq \phi(\lambda) \leq \beta\left(1+|\lambda|^{p}\right)$ for some $\beta>0$ and for all $\lambda \in E$, one 


\section{REFERENCES}

[1] H. Attouch, Variational Convergence for Functions and Operators. Applicable Mathematics Series, Pitman Advanced Publishing Program (1984).

[2] E.J. Balder, Lectures on Young measures theory and its applications in economics. Workshop di Teoria della Misura e Analisi Reale, Grado, 1997, Rend. Istit. Univ. Trieste 31 Suppl. 1 (2000) 1-69.

[3] K. Bhattacharya and R.D. James, A theory of thin films of martinsitic materials with applications to microactuators. J. Mech. Phys. Solids 47 (1999) 531-576.

[4] B. Dacorogna, Direct Methods in the Calculus of Variations. Springer-Verlag, Berlin. Appl. Math. Sciences 78 (1989).

[5] Dal Maso, An introduction to Г-convergence. Birkäuser, Boston (1993).

[6] I. Fonseca, S. Müller and P. Pedregal, Analysis of concentration and oscillation effects generated by gradients. SIAM J. Math. Anal. 29 (1998) 736-756.

[7] L. Freddi and R. Paroni, The energy density of martensitic thin films via dimension reduction. Rapporto di ricerca $\mathrm{n}^{\circ} 9 / 2003$ del dipartimento di Matematica e Informatica dell'Università di Udine.

[8] D. Kinderlehrer and P. Pedregal, Characterization of Young measures generated by gradients. Arch. Rational Mech. Anal. 119 (1991) 329-365.

[9] H. Le Dret and A. Raoult, The nonlinear membrane model as Variational limit in nonlinear three-dimensional elasticity. $J$. Math. Pures Appl., IX. Ser. 74 (1995) 549-578.

[10] P. Pedregal, Parametrized measures and variational Principle. Birkhäuser (1997).

[11] M.A. Sychev, A new approach to Young measure theory, relaxation and convergence in energy. Ann. Inst. Henri Poincaé 16 (1999) $773-812$.

[12] M. Valadier, Young measures. Methods of Nonconvex Analysis, A. Cellina Ed. Springer-Verlag, Berlin. Lect. Notes Math. 1446 (1990) 152-188.

[13] M. Valadier, A course on Young measures. Workshop di Teoria della Misura e Analisi Reale, Grado, September 19-October 2, 1993, Rend. Istit. Mat. Univ. Trieste 26 Suppl. (1994) 349-394 